\title{
Algebraic Aspects of Relatively Pseudocomplemented Posets
}

\author{
Ivan Chajda ${ }^{1} \cdot$ Helmut Länger ${ }^{1,2} \cdot$ Jan Paseka ${ }^{3}$
}

Received: 21 November 2017 / Accepted: 12 April 2019 / Published online: 1 May 2019

(c) The Author(s) 2019

\begin{abstract}
In Chajda and Länger (Math. Bohem. 143, 89-97, 2018) the concept of relative pseudocomplementation was extended to posets. We introduce the concept of a congruence in a relatively pseudocomplemented poset within the framework of Hilbert algebras and we study under which conditions the quotient structure is a relatively pseudocomplemented poset again. This problem is solved e.g. for finite or linearly ordered posets. We characterize relative pseudocomplementation by means of so-called L-identities. We investigate the category of bounded relatively pseudocomplemented posets. Finally, we derive certain quadruples which characterize bounded Hilbert algebras and bounded relatively pseudocomplemented posets up to isomorphism using Glivenko equivalence and implicative semilattice envelope of Hilbert algebras.
\end{abstract}

Keywords Relative pseudocomplementation · Poset · Hilbert algebra · Congruence · Convex poset · Dedekind-MacNeille completion · Glivenko equivalence · Category

Support of the research of all authors by the Austrian Science Fund (FWF), project I 1923-N25, and the Czech Science Foundation (GAČR), project 15-34697L, as well as support of the research of the first two authors by ÖAD, project CZ 02/2019, and support of the research of the first author by IGA, project PřF 2019 015, is gratefully acknowledged.

Jan Paseka

paseka@math.muni.cz

Ivan Chajda

ivan.chajda@upol.cz

Helmut Länger

helmut.laenger@tuwien.ac.at

1 Faculty of Science, Department of Algebra and Geometry, Palacký University Olomouc, 17. listopadu 12, 771 46, Olomouc, Czech Republic

2 Faculty of Mathematics and Geoinformation, Institute of Discrete Mathematics and Geometry, TU Wien, Wiedner Hauptstraße 8-10, 1040, Vienna, Austria

3 Faculty of Science, Department of Mathematics and Statistics, Masaryk University, Kotlářská 2, 611 37, Brno, Czech Republic 


\section{Introduction}

The origin of pseudocomplemented and relatively pseudocomplemented lattices and semilattices can be found in papers quoted in the references in the monographs by H.B. Curry $[17,18]$ and the history of this topic goes back to Skolem as noted in [18], where relatively pseudocomplemented lattices are investigated under the name "implication lattices". Some part of the theory is contained in the paper [1] by R. Balbes. As to pseudocomplemented lattices, they have been investigated formerly by V. Glivenko and M.H. Stone, pseudocomplemented semilattices were introduced and studied by O. Frink [21].

The early work on relative pseudocomplementation in posets was initiated by T. Katriňák $[23,24]$. We cannot omit the paper [33] by S. Rudeanu where he proves that every relatively pseudocomplemented poset is a Hilbert algebra w.r.t. relative pseudocomplementation.

The concept of relative pseudocomplementation plays a crucial role in intuitionistic logic where it models logical implication. Due to this fact, relative pseudocomplementation was investigated by several authors, e.g. by P. Köhler [25] and W. C. Nemitz [29] in their pioneering works. It turns out that relative pseudocomplementation can be extended to posets. A general approach in this direction was already presented by the first two authors in $[7,10]$. An alternative approach was presented by the first author in [6]. The aims of the present paper are as follows:

- to get conditions on a congruence in a relatively pseudocomplemented poset such that the quotient structure of the corresponding Hilbert algebra induced by a congruence is a relatively pseudocomplemented poset again,

- to characterize relative pseudocomplementation by means of so-called L-identities,

- although the class of relatively pseudocomplemented posets does not form a variety, it forms a category whose properties are investigated,

- to introduce the so-called characterizing quadruple which enables us to reconstruct the given bounded relatively pseudocomplemented poset.

At first we recall some basic concepts.

Let $\mathbf{A}=(A, \leq)$ be a non-empty poset and $a, b, c \in A$. For every subset $B$ of $A$ we define

$$
\begin{aligned}
& U(B):=\{y \in A \mid x \leq y \text { for all } x \in B\} \text { and } \\
& L(B):=\{x \in A \mid x \leq y \text { for all } y \in B\} .
\end{aligned}
$$

Then $(U, L)$ is the Galois correspondence induced by $\leq$ and $\bigcap_{i \in I} L\left(B_{i}\right)=L\left(\bigcup_{i \in I} B_{i}\right)$ for any family $B_{i}, i \in I$, of subsets of $A$. If $B=\{a, b\}$, we will write briefly $L(a, b)$ or $U(a, b)$ for $L(\{a, b\})$ or $U(\{a, b\})$, respectively, and $L(a)$ instead of $L(a, a)$.

A subset of a poset $\mathbf{A}$ is up-directed if it is non-empty and every pair of elements has an upper bound in the subset. A poset $\mathbf{A}$ is an up-directed-complete poset if each of its up-directed subsets has a supremum.

If $\mathbf{A}$ has a least element 0 then an element $b$ of $A$ is called the pseudocomplement of $a$ if $b$ is the greatest element $x$ of $(A, \leq)$ satisfying $L(a, x)=\{0\}$. In this case we denote $b$ by $a^{*}$. The poset $\mathbf{A}$ is called pseudocomplemented (or a Boolean ordered set) if each element $a$ of $A$ has a pseudocomplement (and $a^{* *}=a$, respectively). We denote by $B(\mathbf{A})$ the set $\left\{x \in A \mid x=x^{* *}\right\}$ of Boolean elements.

Our study is based on extensively making use of results reached in Hilbert algebras by several authors. Hence, we repeat the basic definitions and concepts. We will use, instead of the classical operation $\rightarrow$ the operation $*$ since our main motivation comes from relatively pseudocomplemented posets. 
A Hilbert algebra $\mathbf{A}=(A, *, 1)$ (see [19, 20, 32-34]) may be treated as a poset $(A, \leq)$ with the greatest element 1 equipped with a binary operation $*$ such that

$$
\begin{gathered}
x * y=1 \text { if and only if } x \leq y, \\
x \leq y * x, \quad x *(y * z) \leq(x * y) *(x * z) .
\end{gathered}
$$

A will be called bounded if it has a smallest element 0 ; in this case, for $x \in A$ we put $x^{*}:=x * 0$.

The class $\mathcal{H}\left(\mathcal{H}_{0}\right)$ of all (bounded) Hilbert algebras considered as algebras of the form $(A, *, 1)((A, *, 1,0)$, respectively) is equationally definable.

Note that a homomorphism of Hilbert algebras is exactly a mapping between Hilbert algebras preserving operations $*$ and 1 (and hence preserving also order).

Since any Boolean algebra is a bounded Hilbert algebra we will further use the notation $(B, *, 1,0)$ for Boolean algebras as well.

An element $c$ of a poset $\mathbf{A}=(A, \leq)$ is called the relative pseudocomplement of a with respect to $b$, in symbols $c=a * b$ ([10]), if $c$ is the greatest element $x$ of $\mathbf{A}$ satisfying $L(a, x) \subseteq L(b)$. If $x * y$ exists for all $x, y \in A$ then the poset $\mathbf{A}$ is called relatively pseudocomplemented and $*$ is said to be a relative pseudocomplementation on $\mathbf{A}$. Relatively pseudocomplemented meet-semilattices are known also as implicative semilattices $[18,29]$, or Brouwerian semilattices.

S. Rudeanu has shown in [33] that the class of all relatively pseudocomplemented posets is a proper subclass of the class of all Hilbert algebras. Hence we may work further in the context of Hilbert algebras.

Definition 1.1 Let $(A, *, 1)$ be a Hilbert algebra. We say that $(A, *, 1)$ is a relatively pseudocomplemented poset if $a * b$ is the greatest element of the set $\{x \in A \mid L(a, x) \subseteq L(b)\}$ for all $a, b \in A$.

Let $\mathcal{R}\left(\mathcal{R}_{0}\right)$ denote the class of all (bounded) relatively pseudocomplemented posets. A homomorphism of (bounded) relatively pseudocomplemented posets is a homomorphism of (bounded) Hilbert algebras.

Obviously, in the case when $\mathbf{A}$ is a meet-semilattice we have that $L(a, x) \subseteq L(b)$ is equivalent to $a \wedge x \leq b$ and hence the concept of relative pseudocomplement in posets is a generalization of the corresponding concept for meet-semilattices introduced in [18].

Definition 1.2 An implicative semilattice is an algebra $(A, \wedge, *, 1)$ of type $(2,2,0)$ such that $(A, \wedge)$ is a meet-semilattice and $(A, *, 1)$ is a relatively pseudocomplemented poset.

Let $\mathcal{I S}\left(\mathcal{I S}_{0}\right)$ denote the class of all (bounded) implicative semilattices. A homomorphism of (bounded) implicative semilattices is a homomorphism of (bounded) Hilbert algebras that preserves finite meets.

We will write $\mathrm{U}_{\mathrm{I}}$ for the forgetful functor from $\mathcal{I} \mathcal{S}$ to $\mathcal{H}$. Fix $\mathbf{A} \in \mathcal{H}$, a pair $(\mathbf{G}, e)$, where $\mathbf{G}$ is an implicative semilattice and $e$ is an injective homomorphism from $\mathbf{A}$ to $\mathrm{U}_{\mathrm{I}}(\mathbf{G})$, is said to be an implicative semilattice envelope of $\mathbf{A}$ if for every $y \in G$ there exists a finite subset $X \subseteq A$ such that $y=\bigwedge e(X)$.

It is well known (see [5]) that there is a functor $\mathrm{S}$ from $\mathcal{H}$ to $\mathcal{I S}$ such that, for any $\mathbf{A} \in \mathcal{H}$, we have an injective homomorphism $e$ from $\mathbf{A}$ to $\mathrm{U}_{\mathrm{I}}(\mathrm{S}(\mathbf{A}))$ and $(\mathrm{S}(\mathbf{A}), e)$ is an implicative semilattice envelope of $\mathbf{A}$. In what follows we will always assume that $A \subseteq \mathrm{S}(\mathbf{A})$, i.e., $\mathbf{A}$ will be a Hilbert subalgebra of $\mathrm{U}_{\mathrm{I}}(\mathrm{S}(\mathbf{A}))$ and $e$ an inclusion. 
The following theorem summarizes some properties from [5], of interest for the present work:

Theorem 1.3 There exists a functor $\mathrm{S}: \mathcal{H} \rightarrow \mathcal{I S}$ that maps every Hilbert algebra $\mathbf{A}$ to an implicative semilattice $\mathbf{S}(\mathbf{A})$, and every homomorphism $h: \mathbf{A} \rightarrow \mathbf{B}$ of Hilbert algebras to a homomorphism $\mathrm{S}(h): \mathrm{S}(\mathbf{A}) \rightarrow \mathrm{S}(\mathbf{B})$ of implicative semilattices such that $\mathrm{S}(h)(\bigwedge X)=$ $\bigwedge h(X)$ for any finite subset $X \subseteq A$. The functor $\mathrm{S}$ is a left adjoint to $\mathrm{U}_{\mathrm{I}}$.

Let $\mathbf{A}=(A, *, 1)$ be a Hilbert algebra. The compatibility relation $\mathrm{C}$ on Hilbert algebras was introduced in [27]. We will use an equivalent definition from [14]: Elements $a, b \in A$ are said to be compatible (in symbols, $a$ b) if they have a lower bound $c$ such that $a \leq b * c$. This lower bound $c$ is necessarily a meet of elements $a$ and $b$; we call also a meet arising in this way compatible. A subset of $A$ is a relative subsemilattice if it is closed under existing compatible meets. To emphasize that the meet of $a$ and $b$ is compatible, it will be written as $a \mathbb{\wedge} b$ (see [15]). $(A, *, 1)$ is an implicative semilattice if and only if all binary meets in $A$ exist and are compatible (see [28, Theorem 11]). If $(\mathbf{G}, e)$ is an implicative semilattice envelope of $\mathbf{A}$ then $e(a \wedge b)=e(a) \wedge e(b)$ for all $a, b \in A$ such that $a$ b.

In what follows we will need several useful properties of Hilbert algebras which can be found, e.g., in [3, 13, 19, 20, 28, 32].

Lemma 1.4 Let $\mathbf{A}=(A, *, 1)$ be a Hilbert algebra. Then the following hold for all $x, y, z \in A$ :

(i) $(A, \leq)$ has a greatest element 1 ,

(ii) $x \leq y$ if and only if $x * y=1$,

(iii) $x * x=x * 1=1$ and $1 * x=x$,

(iv) $x *((x * y) * y)=1$,

(v) $x \leq y$ implies $y * z \leq x * z$,

(vi) $x \leq y$ implies $z * x \leq z * y$,

(vii) $((x * y) * y) * y=x * y$,

(viii) $x *(x * y)=x * y$,

(ix) $y *((x * y) * y)=1$,

(x) $y *(x * y)=1$,

(xi) $x *(y * z)=y *(x * z)$,

(xii) $x *(y * z)=(x * y) *(x * z)$,

(xiii) $\quad(x * y) *((y * x) * x)=(y * x) *((x * y) * y)$.

\section{Congruences}

Let Con A denote the set of all congruences on a Hilbert algebra A. A congruence $\theta$ on a relatively pseudocomplemented poset $\mathbf{A}=(A, *, 1)$ will be the congruence on the Hilbert algebra A. In particular, all results on congruences valid for Hilbert algebras will be also true for relatively pseudocomplemented posets.

Note that an equivalence relation $\theta$ on a poset $(A, \leq)$ is called convex if $a, b, c \in A$, $a \leq b \leq c$ and $(a, c) \in \theta$ together imply $(a, b) \in \theta$, i.e. if every class of $\theta$ is a convex subset of $(A, \leq)$.

Now we can prove 
Theorem 2.1 Let $\mathbf{A}=(A, *, 1)$ be a Hilbert algebra and $\theta \in \mathbf{C o n} \mathbf{A}$. Then $\theta$ is convex and any congruence class of $\theta$ is up-directed.

Proof Let $a, b, c \in A, \theta \in \operatorname{Con}(\mathbf{A})$ and assume that $a<b<c$ and $(a, c) \in \theta$. Since $a<b<c$ we have from Lemma 1.4 (vi) that $(c * a) * a \leq(c * a) * b \leq(c * a) * c$. From Lemma 1.4 (iv) we obtain that $c \leq(c * a) * a$ which yields that $(c * a) * c \leq(c * a) *((c * a) * a)$. Applying Lemma 1.4 (viii) we have $(c * a) *((c * a) * a)=(c * a) * a$. Altogether $(c * a) * a=$ $(c * a) * b$. Hence $a=1 * a=(a * a) * a \theta(c * a) * a=(c * a) * b \theta(a * a) * b=1 * b=b$ according to Lemma 1.4 (iii).

Finally $a, b \in A$ and $a \theta b$ together imply $(a * b) * b \theta(a * a) * a=1 * a=a$ and we have $a, b \leq(a * b) * b$ by Lemma 1.4 (iv) and (ix).

Corollary 2.2 Let $\mathbf{A}=(A, *, 1)$ be a Hilbert algebra that is an up-directed-complete poset and $\theta \in \mathbf{C o n} \mathbf{A}$. Then any congruence class of $\theta$ has a greatest element.

If $\mathbf{A}$ is even a finite Hilbert algebra or a Hilbert algebra satisfying the Ascending Chain Condition then for each $\theta \in \mathbf{C o n} \mathbf{A}$ any congruence class of $\theta$ has a greatest element.

For a Hilbert algebra $\mathbf{A}=(A, *, 1)$ and an equivalence relation $\theta$ on $A$ we define a binary relation $\leq^{\prime}$ on $A / \theta$ as follows:

$$
[x] \theta \leq^{\prime}[y] \theta \text { if and only if there exist } a \in[x] \theta \text { and } b \in[y] \theta \text { with } a * b \theta 1
$$

$(x, y \in A)$. If $\theta \in \operatorname{Con} \mathbf{A}$ we define a binary operation $*^{\prime}$ on $A / \theta$ as follows:

$$
[x] \theta *^{\prime}[y] \theta=[x * y] \theta
$$

$(x, y \in A)$. Moreover,

$$
[x] \theta \leq^{\prime}[y] \theta \text { if and only if }[x] \theta *^{\prime}[y] \theta=[1] \theta
$$

$(x, y \in A)$. From now on, we will write $\bar{x}$ or $\bar{x}_{\theta}$ instead of $[x] \theta$.

In general, if $\theta$ is an equivalence relation on $\mathbf{A}$ then $\leq^{\prime}$ need not be a partial order relation on $A / \theta$, it may be only a quasiorder, i.e., a reflexive and transitive binary relation as will be shown later.

Recall that A. Diego [20] proved that Hilbert algebras form a variety which is locally finite. From the fact that the quotient algebra $\left(A / \theta, *^{\prime}, \overline{1}_{\theta}\right)$ of a Hilbert algebra $(A, *, 1)$ is again a Hilbert algebra and the induced relation $\leq^{\prime}$ on $A / \theta$ coincides with the natural order on the quotient Hilbert algebra we have immediately:

(i) if $a \leq b$ then $\bar{a} \leq^{\prime} \bar{b}$,

(ii) $\left(A / \theta, \leq^{\prime}\right)$ is a poset.

Lemma 2.3 Let $\mathbf{A}=(A, *, 1)$ be a Hilbert algebra, $a, b \in A$ and $\theta \in$ Con $\mathbf{A}$ and assume $\bar{a} \leq \bar{b}$. Then there exists an element $c$ of $\bar{b}$ with $a \leq c$.

Proof Put $c:=(a * b) * b$. Then

$$
c \in \bar{c}=\left(\bar{a} *^{\prime} \bar{b}\right) *^{\prime} \bar{b}=\overline{1} *^{\prime} \bar{b}=\overline{1 * b}=\bar{b}
$$

according to the definition of $\leq^{\prime}$ and Lemma 1.4 (ii). Moreover, we have $a \leq c$ also according to Lemma 1.4 (iv). 
If $\mathbf{A}=(A, *, 1) \in \mathcal{R}$ and $\theta \in \mathbf{C o n} \mathbf{A}$ then $\left(A / \theta, *^{\prime}, \overline{1}_{\theta}\right)$ is a Hilbert algebra satisfying all identities of $(A, *, 1)$. This does not mean that $*^{\prime}$ is the relative pseudocomplementation on $\left(A / \theta, \leq^{\prime}\right)$.

Example 2.4 The poset $\mathbf{A}=(A, \leq)$ with the Hasse diagram

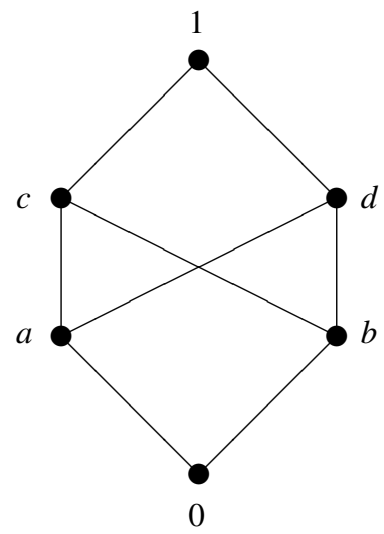

is relatively pseudocomplemented and the operation table of $*$ looks as follows:

\begin{tabular}{l|llllll}
$*$ & 0 & $a$ & $b$ & $c$ & $d$ & 1 \\
\hline 0 & 1 & 1 & 1 & 1 & 1 & 1 \\
$a$ & $b$ & 1 & $b$ & 1 & 1 & 1 \\
$b$ & $a$ & $a$ & 1 & 1 & 1 & 1 \\
$c$ & 0 & $a$ & $b$ & 1 & $d$ & 1 \\
$d$ & 0 & $a$ & $b$ & $c$ & 1 & 1 \\
1 & 0 & $a$ & $b$ & $c$ & $d$ & 1
\end{tabular}

The equivalence relation $\theta:=\{0\}^{2} \cup\{a, c\}^{2} \cup\{b, d\}^{2} \cup\{1\}^{2}$ on $A$ has convex classes, but $\theta \notin$ Con $\mathbf{A}$ since e.g. $(b, d) \in \theta$, but $(b, 1)=(a * b, a * d) \notin \theta$ and $\left(A / \theta, \leq^{\prime}\right)$ is not a poset since $\leq^{\prime}$ is not antisymmetric. On the other hand, $\Phi:=\{0, b\}^{2} \cup\{a, c, d, 1\}^{2} \in$ Con $\mathbf{A}$. Hence $\left(A / \Phi, *^{\prime}, \overline{1}_{\Phi}\right)$ is a Hilbert algebra and $\left(A / \Phi, \leq^{\prime}\right)$ is a poset.

Remark 2.5 Unfortunately, contrary to the class of Hilbert algebras, the class of relatively pseudocomplemented posets is not closed under substructures. Consider e.g. our relatively pseudocomplemented poset $\mathbf{A}=(A, *, 1)$ from Example 2.4 and its subset $S=\{0, c, d, 1\}$. It is immediate that $S$ is closed under $*$. However, this $*$ is not a relative pseudocomplementation in $\mathbf{S}=(S, *, 1)$. Namely, the relative pseudocomplement of $c$ with respect to 0 in $\mathbf{S}$ is $d$ but $d$ differs from $0=c * 0$.

Lemma 2.6 Let $\mathbf{A}=(A, *, 1)$ be a Hilbert algebra. Then $L(a) \cap L(a * b) \subseteq L(b)$ for all $a, b \in A$.

Proof Suppose now $z \in A, z \leq a$ and $z \leq a * b$. Then $z * a=1$ and $z *(a * b)=1$. Using (xii) and (iii) from Lemma 1.4, we have $1=z *(a * b)=(z * a) *(z * b)=1 *(z * b)=z * b$, i.e., $z \leq b$. 
Proposition 2.7 Let $\mathbf{A}=(A, *, 1) \in \mathcal{R}$ and $(C, \leq)$ be a non-empty subchain of $(A, \leq)$ closed under $*$. Then $\mathbf{C}=(C, *, 1) \in \mathcal{R}$.

Proof Let $a, b, x \in C$. Then $a * b \in C$ and $1=a * a \in C$. Since $\mathbf{C}$ is a Hilbert subalgebra of A we have from Lemma 2.6 that $L_{\mathbf{C}}(a) \cap L_{\mathbf{C}}(a * b) \subseteq L_{\mathbf{C}}(b)$. Now, assume that $L_{\mathbf{C}}(a) \cap$ $L_{\mathbf{C}}(x) \subseteq L_{\mathbf{C}}(b)$. We have to consider only the following two cases: If $a \leq x$ then $a \leq b$, i.e., $x \leq 1=a * b$. If $x \leq a$ then $x \leq b \leq a * b$. Hence $a * b$ is the relative pseudocomplement of $a$ with respect to $b$ in $\mathbf{C}$.

Proposition 2.8 Let $\mathbf{A}=(A, *, 1) \in \mathcal{R},(A, \leq)$ be a chain and $\theta \in$ Con $\mathbf{A}$. Then $\left(A / \theta, \leq^{\prime}, *^{\prime}\right) \in \mathcal{R}$ and it is a chain.

Proof Evidently, $\left(A / \theta, *^{\prime}, \overline{1}_{\theta}\right)$ is a Hilbert algebra satisfying $L_{A / \theta}\left(\overline{a * b}_{\theta}\right) \cap L_{A / \theta}\left(\bar{a}_{\theta}\right) \subseteq$ $L_{A / \theta}\left(\bar{b}_{\theta}\right)$ for all $a, b \in A$.

Suppose first that $x, y \in A$. Then $x \leq y$ or $y \leq x$, i.e., $\bar{x}_{\theta} \leq^{\prime} \bar{y}_{\theta}$ or $\bar{y}_{\theta} \leq^{\prime} \bar{x}_{\theta}$. We conclude that $\left(A / \theta, \leq^{\prime}\right)$ is a chain. Assume now that there are elements $a, x, b \in A$ such that $L_{A / \theta}\left(\bar{x}_{\theta}\right) \cap L_{A / \theta}\left(\bar{a}_{\theta}\right) \subseteq L_{A / \theta}\left(\bar{b}_{\theta}\right)$ in the chain $\left(A / \theta, \leq^{\prime}\right)$ and $\bar{x}_{\theta}>^{\prime} \overline{a * b}_{\theta}$. Then $\bar{a}_{\theta}>^{\prime} \bar{b}_{\theta}$ (otherwise we would have $\overline{a * b_{\theta}}=\overline{1}_{\theta}$, a contradiction). If $\bar{x}_{\theta} \geq ' \bar{a}_{\theta}$ then $\bar{x}_{\theta} \geq^{\prime}$ $\bar{a}_{\theta}>^{\prime} \bar{b}_{\theta}$, a contradiction with $\bar{a}_{\theta} \in L_{A / \theta}\left(\bar{x}_{\theta}\right) \cap L_{A / \theta}\left(\bar{a}_{\theta}\right) \subseteq L_{A / \theta}\left(\bar{b}_{\theta}\right)$. Hence $\bar{x}_{\theta}<^{\prime} \bar{a}_{\theta}$. But then we have $\bar{b}_{\theta} \leq^{\prime} \overline{a * b}_{\theta}<\bar{x}_{\theta}$, a contradiction with $\bar{x}_{\theta} \in L_{A / \theta}\left(\bar{x}_{\theta}\right) \cap L_{A / \theta}\left(\bar{a}_{\theta}\right) \subseteq$ $L_{A / \theta}\left(\bar{b}_{\theta}\right)$. We conclude that $\left(A / \theta, *^{\prime}, \overline{1}_{\theta}\right) \in \mathcal{R}$.

Note that an implicative filter $F$ of a Hilbert algebra $\mathbf{A}$ is a non-empty subset of $A$ satisfying $y, y * z \in F$ implies $z \in F$. Denote by Fil $\mathbf{A}$ the set of all implicative filters of $\mathbf{A}$ and, for any subset $B$ of $A$, put $\theta_{B}:=\left\{(x, y) \in A^{2} \mid x * y, y * x \in B\right\}$.

Evidently, if $\Phi \in \mathbf{C o n} \mathbf{A}$ then $\theta_{[1] \Phi}$ is an equivalence relation on $A$. It is easy to see that Con $\mathbf{A}$ and Fil $\mathbf{A}$ form complete lattices with respect to set-inclusion and Fil $\mathbf{A}$ is a Heyting algebra [19]. Then, by the results of Diego [19, 20] which are quoted in Rasiowa [32, Theorem 3.1, Theorem 3.2], one has the following well-known theorem:

Theorem 2.9 Let $\mathbf{A}=(A, *, 1)$ be a Hilbert algebra. Then the mappings $\Phi \mapsto \overline{1}_{\Phi}$ and $F \mapsto \theta_{F}$ are mutually inverse isomorphisms between the lattices (Con $\mathbf{A}, \subseteq$ ) and (Fil $\mathbf{A}, \subseteq$ ).

Now we will work with the useful concept of a nucleus. The definition is as follows:

Definition 2.10 Let $\mathbf{A}=(A, *, 1)$ be a Hilbert algebra. A nucleus on $\mathbf{A}$ is a map $j: A \rightarrow A$ such that for any $a, b \in A$ :

i) $a \leq b$ implies $j(a) \leq j(b)$;

ii) $a \leq j(a)$

iii) $(j \circ j)(a)=j(a)$;

iv) $j(j(a) * j(b))=j(a * b)$.

We put $A_{j}=\{a \in A \mid j(a)=a\}$ and define a binary operation $*_{j}$ on $A_{j}$ by $a *_{j} b=$ $j(a * b)$. Clearly, $A_{j}$ is a subposet of $A$.

Notice that any closure operator on $\mathbf{A}$ which is an endomorphism of $\mathbf{A}$ is a nucleus on $\mathbf{A}$. The identity mapping $\operatorname{id}_{A}$ and the unit mapping $\mathbf{1}: x \mapsto 1$ are examples of nuclei. 
In what follows we will show the converse, i.e., that any nucleus is a closure endomorphism in the sense of [13, Theorem 4.1].

Proposition 2.11 Let $\mathbf{A}=(A, *, 1)$ be a Hilbert algebra and let $j$ be a nucleus on $\mathbf{A}$. Then $j: A \rightarrow A$ is an endomorphism of $\mathbf{A}$ and $*_{j}$ is the restriction of $*$ to $A_{j}$. Moreover, the algebra $\mathbf{A}_{j}=\left(A_{j}, *_{j}, 1\right)$ is a Hilbert algebra which is a subalgebra isomorphic to a quotient algebra of $\mathbf{A}$, and the quotient homomorphism $j: A \rightarrow A_{j}$ is a retract with respect to the inclusion homomorphism $i: A_{j} \rightarrow A$.

Proof Let $a, b \in A$. Clearly, $j(a) * j(b) \leq j(j(a) * j(b))=j(a * b)$. Let us show that $j(a * b) \leq j(a) * j(b)$.

Note first that $j(j(a * b) *(j(a) * b))=j(j(j(a) * j(b)) * j(j(a) * j(b)))=j(1)=1$. We see that $j((j(a * b) *(j(a) * b)) * b)=j(j(j(a * b) *(j(a) * b)) * j(b))=j(1 * j(b))=$ $j(j(b))=j(b)$. Hence $(j(a * b) *(j(a) * b)) * b \leq j(b)$.

Further, from Lemma 1.4 (iv), (xi) and (vi) and the above considerations we have:

$j(a * b) \leq(j(a * b) *(j(a) * b)) *(j(a) * b)=j(a) *((j(a * b) *(j(a) * b)) * b) \leq j(a) * j(b)$.

Assume now $a, b \in A_{j}$. Then $a=j(a)$ and $b=j(b)$. Hence $a * b=j(a) * j(b)=$ $j(a * b)=j(a) *_{j} j(b)=a *_{j} b \in A_{j}$. Moreover, $(j \circ i)(a)=j(a)=a$.

Theorem 2.12 Let $\mathbf{A}=(A, *, 1)$ be a Hilbert algebra.

(i) Let $j$ be a nucleus on $\mathbf{A}$. Then the relation $\theta_{j}$ on A defined by

$$
a \theta_{j} b \text { if and only if } j(a)=j(b),
$$

$a, b \in A$, is a congruence on A. Moreover, any congruence class $\bar{a}_{\theta_{j}}$ has the greatest element $j(a)$.

(ii) Let $\theta$ be a congruence on $\mathbf{A}$ such that any congruence class $\bar{a}_{\theta}$ has a greatest element $\widehat{a}_{\theta}$. Then the mapping $j_{\theta}: A \rightarrow A$ defined by

$$
j_{\theta}(a)=\widehat{a}_{\theta}
$$

for all $a \in A$ is a nucleus on $\mathbf{A}$.

Proof (i) Clearly, the relation $\theta_{j}$ is an equivalence relation on $A$. We have to check that $a \theta_{j} b, c \theta_{j} d$ implies $a * c \theta_{j} b * d$ and $b \leq j(a)$. Let us compute:

$$
j(a * c)=j(a) * j(c)=j(b) * j(d)=j(b * d) .
$$

Hence $\theta_{j}$ is a congruence on A. Since $j(a)=j(j(a))$ we obtain that $a \theta_{j} j(a)$. We have that $b \leq j(b)=j(a)$, i.e., $j(a)$ is the greatest element of the congruence class $\bar{a}_{\theta_{j}}$.

(ii) Clearly, the mapping $j_{\theta}$ is idempotent. Let us show that $j_{\theta}$ is monotone and extensive. Namely, suppose that $a, b \in A$ and $a \leq b$. We want to show that $a \leq j_{\theta}(a) \leq j_{\theta}(b)$. Since $a \theta \widehat{a}_{\theta}$ we have $a \leq \widehat{a}_{\theta}=j_{\theta}(a)$. From $a \leq b$ we have $a * b=1$ which yields that ${\overline{j_{\theta}(a)_{\theta}}} \leq{\overline{j_{\theta}(b)}}_{\theta}$. According to Lemma 2.3 there is an element $c$ of ${\overline{j_{\theta}(b)_{\theta}}}_{\theta}$ with $j_{\theta}(a) \leq c \leq j_{\theta}(b)$.

Let $a, b \in A$. Then $a \theta \widehat{a}_{\theta}, b \theta \widehat{b}_{\theta}$ and $a * b \theta \widehat{a}_{\theta} * \widehat{b}_{\theta}$. Hence $a * b \theta j_{\theta}(a) * j_{\theta}(b)$. We conclude that $j_{\theta}\left(j_{\theta}(a) * j_{\theta}(b)\right)=j_{\theta}(a * b)$, i.e., $j_{\theta}$ is a nucleus. 
Let $\mathbf{A}=(A, *, 1)$ be a Hilbert algebra. We will denote by Conn $\mathbf{A}$ the set of congruences $\theta$ on $\mathbf{A}$ such that any congruence class of $\theta$ has a greatest element. Similarly, we will denote by Nuc $\mathbf{A}$ the set of nuclei $j$ on $\mathbf{A}$. Both sets can be ordered, Conn $\mathbf{A}$ via inclusion of relations and Nuc $\mathbf{A}$ as a subposet of $A^{A}$.

From $[13,16]$ we know that the set Nuc $\mathbf{A}$ is closed under composition $\circ$ and pointwise defined meets. The algebra ( $\left.\operatorname{Nuc} \mathbf{A}, \circ, \wedge, \mathrm{id}_{A}, \mathbf{1}\right)$ is a bounded distributive lattice [13, Corollary 3.5.], in which $\circ$ acts as join and the natural ordering may be defined pointwise.

Theorem 2.13 Let $\mathbf{A}=(A, *, 1)$ be a Hilbert algebra. Then the mappings $\theta \mapsto j_{\theta}$ and $j \mapsto \theta_{j}$ are mutually inverse isomorphisms between the posets (Conn $\mathbf{A}, \subseteq$ ) and (Nuc $\mathbf{A}$, $\leq)$.

Proof Let $a, b \in A, \Phi, \Psi \in$ Conn $\mathbf{A}$ and $k, l \in$ Nuc $\mathbf{A}$. The following are equivalent:

$$
(a, b) \in \theta_{j_{\Phi}}, j_{\Phi}(a)=j_{\Phi}(b), \bar{a}_{\Phi}=\bar{b}_{\Phi},(a, b) \in \Phi
$$

proving $\theta_{j_{\Phi}}=\Phi$. Moreover, the following are equivalent:

$$
j_{\theta_{k}}(a)=b ; b=\widehat{a}_{\theta_{k}} ; k(a)=b
$$

proving $j_{\theta_{k}}=k$. Finally, $\Phi \subseteq \Psi$ implies $j_{\Phi}(a) \leq j_{\Psi}(a)$ for all $a \in A$, and $k \leq l$ implies $\theta_{k} \subseteq \theta_{l}$ completing the proof of the theorem.

From the above theorem and Corollary 2.2 we obtain the following.

Corollary 2.14 Let $\mathbf{A}=(A, *, 1)$ be a Hilbert algebra that is an up-directed-complete poset. Then the poset (Nuc $\mathbf{A}, \leq$ ) is a complete Heyting algebra, Conn $\mathbf{A}=$ Con $\mathbf{A}$, and (Con $\mathbf{A}, \subseteq)$ and (Nuc $\mathbf{A}, \leq$ ) are isomorphic as posets.

Note that Theorem 2.12 and Theorem 2.13 can be derived also by rewording of [13, Theorem 4.1]. Also, Corollary 2.14 implies that for finite Hilbert algebras there is a one-toone correspondence between congruences (filters) and nuclei.

Example 2.15 The algebra $\mathbf{S}=(S, *, 1)$ from Remark 2.5 with the Hasse diagram

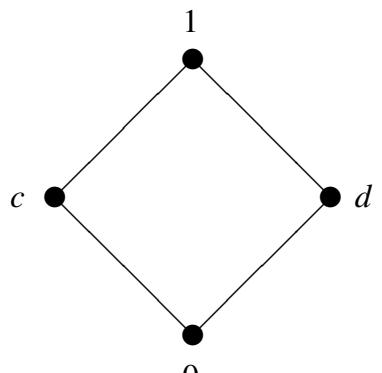


and the operation table of $*$ :

$$
\begin{array}{c|cccc}
* & 0 & c & d & 1 \\
\hline 0 & 1 & 1 & 1 & 1 \\
c & 0 & 1 & d & 1 \\
d & 0 & c & 1 & 1 \\
1 & 0 & c & d & 1
\end{array}
$$

is a Hilbert algebra (since it is a subalgebra of a relatively pseudocomplemented poset, i.e., a Hilbert algebra as well) and it does not belong to $\mathcal{R}$.

Theorem 2.16 Let $\mathbf{A}=(A, *, 1) \in \mathcal{R}$ and $j \in \operatorname{Nuc} \mathbf{A}$. Then $\mathbf{A}_{j}=\left(A_{j}, *_{j}, 1\right) \in \mathcal{R}$.

Proof Since $\left(A_{j}, *_{j}, 1\right)$ is a Hilbert algebra, we obtain from Lemma 2.6 the inclusion $L\left(a, a *_{j} b\right) \cap A_{j} \subseteq L(b) \cap A_{j}$ for all $a, b \in A_{j}$.

Let $a, b, x \in A_{j}$ be such that $L(a, x) \cap A_{j} \subseteq L(b) \cap A_{j}$. Assume that $z \in L(a, x)$. Then $z \leq a$ and $z \leq x$. Hence also $j(z) \leq j(a)=a$ and $j(z) \leq j(x)=x$, i.e., $j(z) \in$ $L(a, x) \cap A_{j} \subseteq L(b) \cap A_{j}$. Therefore $z \leq j(z) \in L(b)$. We see that $L(a, x) \subseteq L(b)$, i.e. $x \leq a * b \leq j(a * b)=a *{ }_{j} b$.

Using the preceding theorem and also Corollary 2.2, we obtain some important sufficient conditions under which the quotient of a relatively pseudocomplemented poset remains relatively pseudocomplemented.

If $\mathbf{A}$ is an up-directed-complete poset or even a finite relatively pseudocomplemented poset then for each $\theta \in$ Con $\mathbf{A}$ the quotient structure is relatively pseudocomplemented and the congruence is generated by some nucleus.

Corollary 2.17 Let $\mathbf{A}=(A, *, 1) \in \mathcal{R}$ and $\theta \in \operatorname{Con} \mathbf{A}$. Then

(i) If all congruence classes of $\theta$ are up-directed-complete posets or finite then $\theta \in$ Conn $\mathbf{A}$ and $\left(A / \theta, *^{\prime}, 1^{\prime}\right) \in \mathcal{R}$.

(ii) If $\mathbf{A}$ is an up-directed-complete poset or finite then $\theta \in \operatorname{Conn} \mathbf{A}$ and $\left(A / \theta, *^{\prime}, 1^{\prime}\right) \in$ $\mathcal{R}$.

Lemma 2.18 Let $\mathbf{A}=(A, \wedge, *, 1)$ be an implicative semilattice and $j$ a nucleus on $(A, *, 1)$. Then $j$ preserves finite meets.

Proof This follows from [5, Theorem 5.1.] and the fact that $j$ is an endomorphism on $(A, *, 1)$.

Proposition 2.19 Let $\mathbf{A}=(A, *, 1)$ be a Hilbert algebra and $j$ a nucleus on $(A, *, 1)$. Then $\mathrm{S}(j): \mathrm{S}(\mathbf{A}) \rightarrow \mathbf{S}(\mathbf{A})$ is a nucleus on $\mathrm{S}(\mathbf{A})$ and $j$ is a restriction of $\mathrm{S}(j)$ to $A$.

Proof Clearly, $\mathrm{S}(j)$ is a homomorphism of implicative semilattices. Since $\mathrm{S}(j) \circ \mathrm{S}(j)=$ $\mathrm{S}(j \circ j)=\mathrm{S}(j)$ we have that $\mathbf{S}(j)$ is idempotent. Let $x \in \mathbf{S}(\mathbf{A})$. Then $x=\bigwedge_{i=1}^{n} a_{i}, a_{i} \in A$. We conclude that $x \leq \bigwedge_{i=1}^{n} j\left(a_{i}\right)=j\left(\bigwedge_{i=1}^{n} a_{i}\right)=j(x)$.

Let $\mathbf{A}=(A, *, 1)$ be a Hilbert algebra, $F \subseteq A$ an implicative filter of $\mathbf{A}$ and $a \in A$. Note that $\mathbf{F}=(F, *, 1)$ is a Hilbert subalgebra of $\mathbf{A}$. We define a mapping $u_{a}^{\mathbf{F}}: F \rightarrow F$ as 
follows:

$$
u_{a}^{\mathbf{F}}(x)=a * x
$$

for all $x \in F$. Since $F$ is an upper set in $\mathbf{A}$ our definition is correct. It is well by known (see e.g. [13]), that applying Lemma 1.4 (xii) we obtain that $u_{a}^{\mathbf{F}}$ is an endomorphism of $\mathbf{F}$. We then define the following relation $\theta_{a}^{\mathbf{F}}$ on $\mathbf{F}$ :

$$
x \theta_{a}^{\mathbf{F}} y \text { if and only if } u_{a}^{\mathbf{F}}(x)=u_{a}^{\mathbf{F}}(y)
$$

for all $x, y \in F$. If $A=F$ we put $\theta_{a}=\theta_{a}^{\mathbf{A}}$.

Lemma 2.20 Let $\mathbf{A}=(A, *, 1)$ be a Hilbert algebra, $F \subseteq A$ an implicative filter of $\mathbf{A}$ and $a \in A$. Then $\theta_{a}^{\mathbf{F}} \in \mathbf{C o n n} \mathbf{F}$ and $[1] \theta_{a}^{\mathbf{F}}=U(a) \cap F=\{x \in F \mid x \geq a\}$.

Proof From Lemma 1.4 (x), (vi) and (viii) we obtain that $u_{a}^{\mathbf{F}}$ is extensive, monotone and idempotent. Since $u_{a}^{\mathbf{F}}$ is an endomorphism of $\mathbf{F}$ we have that $u_{a}^{\mathbf{F}}$ is a nucleus on $\mathbf{F}$ and hence $\theta_{a}^{\mathbf{F}} \in \operatorname{Conn} \mathbf{F}$.

For $x \in F$ we have $x \in \overline{1}_{\theta_{a}^{\mathbf{F}}}$ if and only if $a * x=a * 1=1$ if and only if $x \in$ $U(a) \cap F$.

Corollary 2.21 Let $\mathbf{A}=(A, *, 1) \in \mathcal{R}, a \in A$. Then $\left(A / \theta_{a}, *^{\prime}, 1^{\prime}\right) \in \mathcal{R}$.

In what follows we show that $\theta_{a}$ is in fact a principal congruence and that every principal congruence on a Hilbert algebra is of this form.

Lemma 2.22 Let $\mathbf{A}=(A, *, 1)$ be a Hilbert algebra, $a \in A$. Then $\theta_{a}=\theta(a, 1)$, i.e., it is the congruence generated by the pair $(a, 1)$.

Proof Since $a \theta_{a} 1$ we have that $\theta(a, 1) \subseteq \theta_{a}$.

Let us check that $\theta(a, 1) \supseteq \theta_{a}$. Assume that $x \theta_{a} y$. Since $1 \theta(a, 1) a$ we have that $x=1 * x \theta(a, 1) a * x$. Similarly, $y \theta(a, 1) a * y$. But $a * x=a * y$ which yields $x \theta(a, 1) y$. Therefore $\theta(a, 1) \supseteq \theta_{a}$.

Lemma 2.23 Let $\mathbf{A}=(A, *, 1)$ be a Hilbert algebra, $a, b \in A, a \leq b$. Then $\theta(b * a, 1)=$ $\theta(a, b)$.

Proof Clearly, $b * a \theta(a, b)$ 1, i.e., $\theta(b * a, 1) \subseteq \theta(a, b)$. Conversely, $b * a \theta(b * a, 1) 1$. We see that $(b * a) * a \theta(b * a, 1) 1 * a=a$ and $a \leq b \leq(b * a) * a$. Since $\theta(b * a, 1)$ is convex we have that $a \theta(b * a, 1) b$, i.e., $\theta(a, b) \subseteq \theta(b * a, 1)$.

\section{The Dedekind-MacNeille Completion and Generalized Glivenko Equivalences}

It is well-known that every poset $(A, \leq)$ can be embedded into a complete lattice $\mathbf{L}$. We frequently take the so-called Dedekind-MacNeille completion $\operatorname{DM}(A, \leq)$ for this $\mathbf{L}$. The question arises if also every relatively pseudocomplemented poset can be embedded into a complete relatively pseudocomplemented lattice, i.e., a complete Heyting algebra. 
In the sequel we show that in fact the Dedekind-MacNeille completion of a relatively pseudocomplemented poset is again relatively pseudocomplemented.

Hence, let $\mathbf{A}=(A, \leq)$ be a poset. Put $\operatorname{DM}(\mathbf{A}):=\{B \subseteq A \mid L U(B)=B\}$. (We simply write $L U(B)$ instead of $L(U(B))$. Analogous simplifications are used in the sequel.) Then $\operatorname{DM}(\mathbf{A})=\{L(B) \mid B \subseteq A\}, \mathbf{D M}(\mathbf{A}):=(\operatorname{DM}(\mathbf{A}), \subseteq)$ is a complete lattice and $x \mapsto L(x)$ is an embedding from $\mathbf{A}$ to $\mathbf{D M}(\mathbf{A})$ preserving all existing joins and meets, and an order isomorphism between $\mathbf{A}$ and $(\{L(x) \mid x \in A\}, \subseteq)$.

Let $\mathbf{A}$ be equipped with a binary operation $*$. We introduce a new operation $*$ on $\operatorname{DM}(\mathbf{A})$ as follows:

$$
X * Y:=\bigcap_{a \in X, b \in U(Y)} L(a * b)
$$

for all $X, Y \in \mathrm{DM}(\mathbf{A})$.

Theorem 3.1 Let $\mathbf{A}=(A, \leq)$ be a poset and $*$ a binary operation on A. Then the following are equivalent:

(i) A has the top element 1 and $(A, *, 1)$ is a relatively pseudocomplemented poset,

(ii) $(\mathrm{DM}(\mathbf{A}), *, A)$ is a relatively pseudocomplemented poset and

$$
L(x) * L(y)=L(x * y)
$$

for every $x, y \in A$.

Proof (i) $\Rightarrow$ (ii): The implication (i) $\Rightarrow$ (ii) is already known, see Y. S. Pawar [31].

(ii) $\Rightarrow$ (i): Clearly, $\mathbf{A}$ has the top element 1, i.e., $A=L(1)$. For $a, b, c \in A$ the following are equivalent: $L(a, c) \subseteq L(b), L(a) \cap L(c) \subseteq L(b), L(c) \subseteq L(a) * L(b)$, $L(c) \subseteq L(a * b), c \leq a * b$. Hence $(A, *, 1)$ is relatively pseudocomplemented.

In the theory of pseudocomplemented lattices, a prominent role is played by so-called Glivenko equivalences, see e.g. [1]. Of course, if a relative pseudocomplemented lattice has a least element 0 then for each element $x$ the relative pseudocomplement with respect to 0 is the pseudocomplement of $x$.

It is worth noticing that Glivenko-Frink theorems for Hilbert algebras were discussed by D. Busneag [4] and S. Rudeanu [34].

Let $\mathbf{A}=(A, *, 1)$ be a Hilbert algebra and $a \in A$. We define a mapping $\gamma_{a}^{\mathbf{A}}: A \rightarrow A$ as follows:

$$
\gamma_{a}^{\mathbf{A}}(x)=(x * a) * a
$$

for all $x \in A$. We then define the so-called generalized Glivenko equivalence $\Gamma_{a}^{\mathbf{A}}$ on $A$ by

$$
(x, y) \in \Gamma_{a}^{\mathbf{A}} \text { if and only if } x * a=y * a \text { if and only if } \gamma_{a}^{\mathbf{A}}(x)=\gamma_{a}^{\mathbf{A}}(y)
$$

$(x, y \in A)$. Note that we used here Lemma 1.4 (vii).

If $\mathbf{A}$ is a bounded Hilbert algebra we call $\Gamma_{0}^{\mathbf{A}}$ the Glivenko equivalence. Similarly as for lattices, the Glivenko equivalence $\Gamma_{0}^{\mathbf{A}}$ on a bounded Hilbert algebra $\mathbf{A}$ can be defined by

$$
(x, y) \in \Gamma_{0}^{\mathbf{A}} \text { if and only if } x^{*}=y^{*}
$$

$(x, y \in A)$. The question arises when $\Gamma_{a}^{\mathbf{A}} \in \mathrm{Con} \mathbf{A}$. The following example shows such a case:

Example 3.2 Consider the relatively pseudocomplemented poset $\mathbf{A}=(A, *, 1)$ from Example 2.4. Then $\Psi:=\{0\}^{2} \cup\{a\}^{2} \cup\{b\}^{2} \cup\{c, d, 1\}^{2}$ is the Glivenko equivalence on $A$. It is 
evident that $\Psi \in$ Con $\mathbf{A}$ and $\left(A / \Psi, *^{\prime}, 1^{\prime}, 0^{\prime}\right)$ is even a four-element Boolean algebra with complementation *.

This example is not exceptional, as S. Rudeanu was able to show in [34, Theorem 2.2]. On the contrary, it turns out that the quotient is always a Boolean algebra.

Theorem 3.3 Let $\mathbf{A}=(A, *, 1)$ be a Hilbert algebra, $a \in A$ and $\Gamma_{a}^{\mathbf{A}}$ be its generalized Glivenko equivalence. Then $\left(A / \Gamma_{a}^{\mathbf{A}}, *^{\prime}, 1^{\prime}, 0^{\prime}\right)$ is a Boolean algebra and $\gamma_{a}^{\mathbf{A}}(x * y)=$ $\gamma_{a}^{\mathbf{A}}(x) * \gamma_{a}^{\mathbf{A}}(y)$ for all $x, y \in A, y \geq a$.

We denote by $B_{a}(\mathbf{A})$ the set $\left\{x \in A \mid \gamma_{a}^{\mathbf{A}}(x)=x\right\}$. Clearly, $\left(B_{a}(\mathbf{A}), *, 1\right)$ is a bounded Hilbert algebra with smallest element $a$ which is a subalgebra of $\mathbf{A}$.

Corollary 3.4 Let $\mathbf{A}=(A, *, 1)$ be a Hilbert algebra, $a \in A$. Then $\left(B_{a}(\mathbf{A}), *, 1\right) \in \mathcal{R}$.

Though in general $\Gamma_{a}^{\mathbf{A}} \notin \operatorname{Con} \mathbf{A}$ (see [26] for Heyting algebras) we have from [34, Theorem 2.2] and [13, Theorem 4.1] the following:

Corollary 3.5 Let $\mathbf{A}=(A, *, 1)$ be a bounded Hilbert algebra with smallest element 0 . Then $\gamma_{0}^{\mathbf{A}}: A \rightarrow A$ is an endomorphism of $\mathbf{A}$ such that the identity $x * \gamma_{0}^{\mathbf{A}}(y)=$ $\gamma_{0}^{\mathbf{A}}(x) * \gamma_{0}^{\mathbf{A}}(y)$ is satisfied, $\gamma_{0}^{\mathbf{A}} \in \operatorname{Nuc} \mathbf{A}, \Gamma_{0}^{\mathbf{A}} \in \operatorname{Conn} \mathbf{A}$ and $\left(A / \Gamma_{0}^{\mathbf{A}}, *^{\prime}, 1^{\prime}, 0^{\prime}\right)$ is a Boolean algebra.

In what follows, we shall denote, for a bounded Hilbert algebra $\mathbf{A}$ with smallest element 0 , by $B(\mathbf{A})$ the Boolean algebra $\left(B_{0}(\mathbf{A}), *, 1,0\right)$.

Corollary 3.6 Let $\mathbf{A}=(A, *, 1,0)$ be a bounded Hilbert algebra. Then the mapping ${ }^{* *}: A \rightarrow B(\mathbf{A})$ and the embedding $i_{B(\mathbf{A})}: B(\mathbf{A}) \rightarrow A$ are homomorphisms of bounded Hilbert algebras such that ${ }^{* *} \circ i_{B(\mathbf{A})}=i d_{B(\mathbf{A})}$.

Note that Corollary 3.6 is essentially Theorem 5.2.24 of [4]. We will now state the following useful lemmas and their corollaries:

Lemma 3.7 Let $\mathbf{A}=(A, *, 1,0)$ be a bounded Hilbert algebra, $x \in A$. Then $x^{* *} \wedge\left(x^{* *} * x\right)$ exists, $x=x^{* *} \wedge\left(x^{* *} * x\right), x^{*} \leq x^{* *} * x, x^{* *} *\left(x^{* *} * x\right)=x^{* *} * x$ and $\left(x^{* *} * x\right)^{*}=0$. Moreover, if $d \in A$ such that $x^{* *} \wedge d$ exists, $x=x^{* *} \wedge d$ and $x^{* *} * d=d$ then $d=x^{* *} * x$.

Proof Clearly, $x \leq x^{* *}$ and $x \leq x^{* *} * x$. Hence $x$ is a lower bound of $x^{* *}$ and $x^{* *} * x$. Let $y \in A$ be some lower bound of $x^{* *}$ and $x^{* *} * x$. From Lemma 1.4 (xii) and (iii) we have $1=y *\left(x^{* *} * x\right)=\left(y * x^{* *}\right) *(y * x)=1 *(y * x)=y * x$, whence $y \leq x$. We conclude that $x=x^{* *} \wedge\left(x^{* *} * x\right)$.

Also, we have $x^{*} \wedge x^{* *}=0 \leq x$, i.e., $x^{*} \leq x^{* *} * x$. Therefore $\left(x^{* *} * x\right)^{*} \leq x^{* *}$ and $\left(x^{* *} * x\right)^{*} \leq x^{*}$, i.e., $\left(x^{* *} * x\right)^{*}=0=x^{*} \wedge x^{* *}$.

From Lemma 1.4 (viii) we obtain that $x^{* *} *\left(x^{* *} * x\right)=x^{* *} * x$.

Let $d \in A$ satisfy the assumptions of our lemma. Then $x \leq d$ and $x^{* *} * x \leq x^{* *} * d=d$. As the meet in $x=x^{* *} \wedge d$ is compatible, the reverse inequality, $d \leq x^{* *} * x$, also holds. 
The following corollary follows directly from the definition of $C$.

Corollary 3.8 Let $\mathbf{A}=(A, *, 1,0)$ be a bounded Hilbert algebra, $x \in$ A. Then $x^{* *} C$ $x^{* *} * x$ and $x=x^{* *} \mathbb{\wedge}\left(x^{* *} * x\right)$.

Lemma 3.9 Let $\mathbf{A}=(A, *, 1,0)$ be a bounded Hilbert algebra, $x, y \in A$. Then

(i) $x^{* *} *\left[\left(x^{* *} * x\right) *\left(y^{* *} * y\right)\right]=\left(x^{* *} * y^{* *}\right) *(x * y)=(x * y)^{* *} *(x * y)$,

(ii) $x * y=\left(x^{* *} * y^{* *}\right) \wedge\left(x^{* *} *\left[\left(x^{* *} * x\right) *\left(y^{* *} * y\right)\right]\right)$

(iii) $x \leq y$ if and only if $x^{* *} \leq y^{* *}$ and $x^{* *} \leq\left(x^{* *} * x\right) *\left(y^{* *} * y\right)$.

Proof (i): Let us compute:

$$
\begin{aligned}
x^{* *} *\left[\left(x^{* *} * x\right) *\left(y^{* *} * y\right)\right] & =\left[x^{* *} *\left(x^{* *} * x\right)\right] *\left[x^{* *} *\left(y^{* *} * y\right)\right] \\
& =\left(x^{* *} * x\right) *\left[x^{* *} *\left(y^{* *} * y\right)\right] \\
& =x^{* *} *\left[x *\left(y^{* *} * y\right)\right]=x^{* *} *\left[y^{* *} *(x * y)\right] \\
& =\left(x^{* *} * y^{* *}\right) *\left[x^{* *} *(x * y)\right]=\left(x^{* *} * y^{* *}\right) *(x * y) .
\end{aligned}
$$

The first, third and fifth equality follow from Lemma 1.4 (xii), the second one from Lemma 1.4 (viii), the fourth one from Lemma 1.4 (xi). The last equality follows from the fact that $x * y \leq x^{* *} *(x * y) \leq x *(x * y)=x * y$; the first inequality follows from Lemma $1.4(\mathrm{x})$, the second one from Lemma $1.4(\mathrm{v})$ and the equality follows from Lemma 1.4 (viii). Since $x^{* *} * y^{* *}=(x * y)^{* *}$ we obtain the remaining part of the statement.

(ii): We have from Lemma 3.7 and (i):

$x * y=(x * y)^{* *} \wedge\left((x * y)^{* *} *(x * y)\right)=\left(x^{* *} * y^{* *}\right) \wedge\left(x^{* *} *\left[\left(x^{* *} * x\right) *\left(y^{* *} * y\right)\right]\right)$.

(iii): Evidently, from (ii) we obtain

$$
\begin{aligned}
x \leq y & \Longleftrightarrow x * y=1 \Longleftrightarrow x^{* *} * y^{* *}=1 \text { and } x^{* *} *\left[\left(x^{* *} * x\right) *\left(y^{* *} * y\right)\right]=1 \\
& \Longleftrightarrow x^{* *} \leq y^{* *} \text { and } x^{* *} \leq\left(x^{* *} * x\right) *\left(y^{* *} * y\right) .
\end{aligned}
$$

Corollary 3.10 Let $\mathbf{A}=(A, *, 1,0)$ be a bounded Hilbert algebra, $x \in A, y \in B(\mathbf{A})$ such that $x^{* *}=1$. Then $x * y=y$.

Proof Using Lemma 3.9 part (ii) and the fact that $x^{* *}=1$ and $y^{* *}=y$ we have

$$
x * y=(1 * y) \wedge(1 *(x *(y * y)))=y \wedge(1 *(x * 1))=y \wedge(1 * 1)=y .
$$

Lemma 3.11 Let $\mathbf{A}=(A, *, 1) \in \mathcal{R}, a, b \in A$. Then $\gamma_{\gamma_{a}^{\mathbf{A}}\left(\gamma_{b}^{\mathbf{A}}(a)\right)}^{\mathbf{A}}=\gamma_{\gamma_{b}^{\mathbf{A}}\left(\gamma_{a}^{\mathbf{A}}(b)\right)}^{\mathbf{A}}$.

Proof Let us consider the mappings $\gamma_{L(a)}^{\mathbf{D M}(\mathbf{A})}, \gamma_{L(b)}^{\mathbf{D M}(\mathbf{A})}: \operatorname{DM}(\mathbf{A}) \rightarrow \operatorname{DM}(\mathbf{A})$. Then they are modal operators on the Heyting algebra $(\operatorname{DM}(\mathbf{A}), *, A)$ in the sense of the paper 
[26, Theorem 1.3 and Lemma 1.4]. Moreover, from [26, Lemma 3.5 and Lemma 3.6] we obtain that the join of these modal operators in the lattice $(M(\mathbf{D M}(\mathbf{A})), \leq)$ of all modal operators exists and $\gamma_{L(a)}^{\mathbf{D M}(\mathbf{A})} \vee \gamma_{L(b)}^{\mathbf{D M}(\mathbf{A})}=\gamma_{\gamma_{L(a)}^{\mathbf{D M}(\mathbf{A})}\left(\gamma_{L(b)}^{\mathbf{D M}(\mathbf{A})}(L(a))\right)}^{\mathbf{D M}(\mathbf{A})}$. Similarly, $\gamma_{L(b)}^{\mathbf{D M}(\mathbf{A})} \vee$ $\gamma_{L(a)}^{\mathbf{D M}(\mathbf{A})}=\gamma_{\gamma_{L(b)}^{\mathbf{D M}(\mathbf{A})}\left(\gamma_{L(a)}^{\mathbf{D M}(\mathbf{A})}(L(b))\right)}^{\mathbf{D M}(\mathbf{A})}$. We conclude $\gamma_{\gamma_{L(a)}^{\mathbf{D M}(\mathbf{A})}\left(\gamma_{L(b)}^{\mathbf{D M}(\mathbf{A})}(L(a))\right)}^{\mathbf{D M}(\mathbf{A})}=\gamma_{\gamma_{L(b)}^{\mathbf{D M}(\mathbf{A})}\left(\gamma_{L(a)}^{\mathbf{D M}(\mathbf{A})}(L(b))\right)}^{\mathbf{D M}(\mathbf{A})}$. If we identify $a$ with $L(a)$ we obtain that $\gamma_{\gamma_{a}^{\mathbf{A}}\left(\gamma_{b}^{\mathbf{A}}(a)\right)}^{\mathbf{A}}=\gamma_{\gamma_{b}^{\mathbf{A}}\left(\gamma_{a}^{\mathbf{A}}(b)\right)}^{\mathbf{A}}$.

Corollary 3.12 Let $\mathbf{A}=(A, *, 1) \in \mathcal{R}$ and $a, b \in A$. Then $\gamma_{a}^{\mathbf{A}}\left(\gamma_{b}^{\mathbf{A}}(a)\right)=\gamma_{b}^{\mathbf{A}}\left(\gamma_{a}^{\mathbf{A}}(b)\right)$.

The following proposition is well known:

Proposition 3.13 [14, Proposition 2.2.] An algebra $\mathbf{A}=(A, *, 1)$ is a Hilbert algebra if and only if it is a subreduct of an implicative semilattice.

Using the above proposition we obtain the following corollary:

Corollary 3.14 Let $\mathbf{A}=(A, *, 1)$ be a Hilbert algebra and $a, b \in$ A. Then

$$
(((a * b) * b) * a) * a=(((b * a) * a) * b) * b .
$$

Proof Since a Hilbert algebra $\mathbf{A}$ is a subreduct of an implicative semilattice $\mathbf{C}$ and $\mathbf{C} \in \mathcal{R}$ the result follows from Corollary 3.12 .

Our next task is to characterize relative pseudocomplementation by some kind of identities. It is well-known (see e.g. [1]) that for lattices or meet-semilattices this is possible. Because the class of relatively pseudocomplemented posets is not closed under substructures, one cannot expect that it could be characterized by means of identities only. The identities having sets of the form $L(B), U(B)$ for subsets $B$ of the poset $\mathbf{A}$ on both sides are called $L U$-identities, see e.g. [11]. In the case when we work only with subsets of the form $L(B)$ we speak about $L$-identities. Using this machinery, we can prove the following:

Theorem 3.15 Let $\mathbf{A}=(A, \leq)$ be a poset with a greatest element 1 and $*$ a binary operation on $A$. Then $*$ is a relative pseudocomplementation on $\mathbf{A}$ if and only if it satisfies the following L-identities:

(a) $L(x, x * y) \approx L(x, y)$,

(b) $L(x * y, y) \approx L(y)$,

(c) $L(x) * L(x, y) \approx L(x * y)$,

Proof Let $a, b, c \in A$. Assume $\mathbf{A}$ to be a relatively pseudocomplemented poset. From Theorem 3.1 we obtain that $(\operatorname{DM}(\mathbf{A}), *, A)$ is a complete relatively pseudocomplemented lattice such that $L(a) * L(b)=L(a * b)$. In particular, (a), (b) and (c) follow from [9, Theorem 4.2.1]. 
Conversely, assume A to satisfy (a)-(c). Assume $c \leq a * b$. Then $L(a, c) \subseteq L(a, a * b)=$ $L(a, b) \subseteq L(b)$ according to (a). Conversely, assume that $L(a, c) \subseteq L(b)$. From (c) we conclude $L(a) * L(a, b)=L(a * b)$ and $L(a) * L(a, c)=L(a * c)$. Now we have

$$
\begin{aligned}
L(a * c) & =L(a) * L(a, c)=L(a) * L(a, c, b)=\bigcap_{d \in L(a), e \in U(L(a, c, b))} L(d * e) \subseteq \\
& \subseteq \bigcap_{d \in L(a), e \in U(L(a, b))} L(d * e)=L(a) * L(a, b)=L(a * b) .
\end{aligned}
$$

Hence $a * c \leq a * b$. Since $c \leq a * c$ by (b), we conclude that $c \leq a * b$. Together, $a * b$ is the relative pseudocomplement of $a$ with respect to $b$.

Theorem 3.16 Let $\mathbf{A}=(A, *, 1)$ be a Hilbert algebra. Then $\mathbf{A}$ is a relatively pseudocomplemented poset if and only if it satisfies the L-identity $L(x) * L(x, y) \approx L(x *$ $y)$.

Proof By Theorem 3.15, every relatively pseudocomplemented poset is a Hilbert algebra which satisfies $L$-identity (c) of Theorem 3.15 .

Conversely, assume that a Hilbert algebra $(A, *, 1)$ satisfies the mentioned $L$-identity. By Proposition 2.6 we have $L(a) \cap L(a * b) \subseteq L(b)$ for all $a, b \in A$. Assume now $L(a) \cap$ $L(x) \subseteq L(b)$ for some $x \in A$. Then, by the $L$-identity, $L(a * x)=L(a) * L(a, x)=$ $L(a) * L(a, x, b) \subseteq L(a) * L(a, b)=L(a * b)$; thus $L(a * x) \subseteq L(a * b)$, i.e., $x \leq a * x \leq a * b$. Hence $a * b$ is the relative pseudocomplement of $a$ with respect to $b$ in $\mathbf{A}$.

\section{The Category of Bounded Relatively Pseudocomplemented Posets}

In what follows, we will study the category $\mathcal{R}_{0}$ whose objects are bounded relatively pseudocomplemented posets and morphisms are mappings preserving the bottom element, the top element and the binary operation $*$. Note that the category of Heyting algebras is a subcategory of $\mathcal{R}_{0}$ but it is not full since there are mappings between Heyting algebras which are morphisms of bounded relatively pseudocomplemented posets but they do not preserve the lattice operations.

Since $\mathcal{R}$ is a full subcategory of the category of Hilbert algebras and similarly $\mathcal{R}_{0}$ is a full subcategory of the category of bounded Hilbert algebras one can use the already obtained results in this broader context [4, 22].

It is well known that the category of Boolean algebras forms a reflective subcategory of the category of bounded relatively pseudocomplemented lattices, i.e., Heyting algebras and that complete Boolean algebras are injective objects in Heyting algebras. Our goal is to show that similar results can be proved also for the category of bounded relative pseudocomplemented posets. A related result was obtained in [4, Theorem 5.5.1, Theorem 5.5.2] where the author proved that in the category of bounded Hilbert algebras any injective object is a complete Boolean algebra, and the complete Boolean algebras are injective objects.

Recall that a monomorphism is a morphism $m$ such that $m \circ f=m \circ g$ implies $f=g$. A morphism $f: A \rightarrow B$ is an isomorphism if there is a morphism $g: B \rightarrow A$ such that $f \circ g=\mathrm{id}_{B}$ and $g \circ f=\mathrm{id}_{A}$. The morphism $g$ is uniquely determined, usually denoted by $f^{-1}$, and called the inverse of $f$. 
Proposition 4.1 Let $m: \mathbf{A} \rightarrow \mathbf{B}$ be a morphism in $\mathcal{R}$. The following are equivalent:

(i) $m$ is injective.

(ii) $m$ is a monomorphism.

(iii) $m$ satisfies $m(x)=1$ implies $x=1$.

Proof (i) $\Longrightarrow$ (ii): An injective morphism $m$ is obviously a monomorphism: if $m(f(x))=m(g(x))$ then we have $f(x)=g(x)$.

(ii) $\Longrightarrow$ (iii): Now let $m: \mathbf{A} \rightarrow \mathbf{B}$ be a monomorphism in $\mathcal{R}$ such that $m(x)=1=m(1)$ for some $x \in A$. Consider the morphisms $u_{x}^{\mathbf{A}}, \mathrm{id}_{A}: \mathbf{A} \rightarrow \mathbf{A}$ in $\mathcal{R}$. Then clearly $m \circ u_{x}^{\mathbf{A}}=m \circ \mathrm{id}_{A}$, i.e., $u_{x}^{\mathbf{A}}=\mathrm{id}_{A}$. We conclude that $x=\operatorname{id}_{A}(x)=x * x=1$.

(iii) $\Longrightarrow$ (i): Let $m(x)=m(y)$ for some $x, y \in A$. Then $1=m(x) * m(y)=m(x * y)$, i.e., $x * y=1$. We have that $x \leq y$ and similarly $y \leq x$. Hence $x=y$, i.e., $m$ is injective.

Recall also that in any bounded relatively pseudocomplemented poset $\mathbf{A}$ each element $x$ of $\mathbf{A}$ has its pseudocomplement $x^{*}=x * 0$.

Note that we have from Corollary 3.6 that the mapping **: $A \rightarrow B(\mathbf{A})$ is a morphism of bounded relatively pseudocomplemented posets. We will call it the canonical mapping.

Lemma 4.2 Let $\mathbf{A}=(A, *, 1,0) \in \mathcal{R}_{0}$ and let $\mathbf{B}=(B, *, 1,0) \in \mathcal{R}_{0}$ be a Boolean algebra. Let $h: \mathbf{A} \rightarrow \mathbf{B}$ be a morphism of bounded relatively pseudocomplemented posets. Then there exists a uniquely determined Boolean homomorphism $\bar{h}: B(\mathbf{A}) \rightarrow \mathbf{B}$ such that the following diagrams commute:
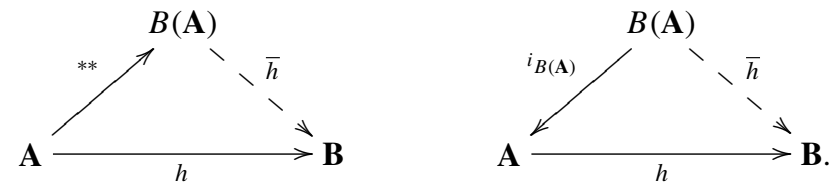

Proof Since $h\left(x^{*}\right)=h(x)^{*}$ we obtain that $h\left(x^{* *}\right)=h(x)$ for all $x \in A$. Let $\bar{h}$ be the restriction of $h$ to $B(\mathbf{A})$. Since join, meet and negation in $B(\mathbf{A})$ can be expressed using $*$ and 0 only, we have that $\bar{h}$ is a Boolean homomorphism and $\bar{h}\left(x^{* *}\right)=h(x)$ for all $x \in A$ (which ensures the uniqueness of $\bar{h}$ ).

Lemma 4.3 Let $\mathbf{A}=(A, *, 1,0) \in \mathcal{R}_{0}$ and let $\mathbf{C}=(C, *, 1,0) \in \mathcal{R}_{0}$. Let $h: \mathbf{A} \rightarrow \mathbf{C}$ be a morphism of bounded relatively pseudocomplemented posets. Then there exists a uniquely determined Boolean homomorphism $\bar{h}: B(\mathbf{A}) \rightarrow B(\mathbf{C})$ such that the following diagrams commute:
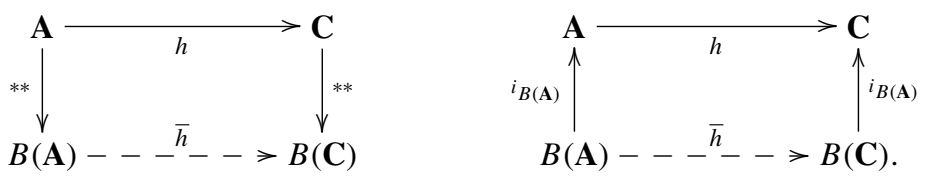

If $h$ is one-to-one, so is $\bar{h}$. 
Proof The existence and the uniqueness of $\bar{h}$ follow from Lemma 4.2. Let us assume that $h$ is one-to-one and that $\bar{h}(x)=\bar{h}(y)$. Then $h(x)=h(y)$ since $x, y \in B(\mathbf{A})$ and $h(x), h(y) \in$ $B(\mathbf{C})$. We conclude $x=y$.

Proposition 4.4 Let $m: \mathbf{A} \rightarrow \mathbf{B}$ be a morphism in $\mathcal{R}_{0}$. The following are equivalent:

(i) $m$ is injective.

(ii) $m$ is a monomorphism in $\mathcal{R}_{0}$.

(iii) $m$ satisfies $m(x)=1$ implies $x=1$.

Proof (i) $\Longrightarrow$ (ii) and (iii) $\Longrightarrow$ (i) follow by the same considerations as in Proposition 4.1.

(ii) $\Longrightarrow$ (iii): Let $m: \mathbf{A} \rightarrow \mathbf{B}$ be a monomorphism in $\mathcal{R}_{0}$ such that $m(x)=1=m(1)$ for some $x \in A$. From Lemma 3.7 we know that $x=x^{* *} \wedge d$ for some $d \in A$ with $d^{*}=0$. Therefore $m\left(x^{* *}\right)=m(d)=1$. Let us show that $x^{* *}=d=1$. From Corollary 3.4 we know that $(B(\mathbf{A}), *, 1,0) \in \mathcal{R}_{0}$. Let us put $T=\left\{0, x^{*}, x^{* *}, 1\right\}$. Then also $(T, *, 1,0) \in \mathcal{R}_{0}$ and the inclusion map $i_{T}: T \rightarrow A$ is a morphism in $\mathcal{R}_{0}$. Let us define a mapping $g: T \rightarrow A$ by $g(0)=g\left(x^{*}\right)=0$ and $g(1)=g\left(x^{* *}\right)=1$. One can easily verify that $g$ is a morphism in $\mathcal{R}_{0}$ and that $m \circ g=m \circ i_{T}$. Hence $g=i_{T}$, i.e., $x^{* *}=i_{T}\left(x^{* *}\right)=g\left(x^{* *}\right)=1$.

Now, let us put $C=\{0, d, 1\}$. Then $C$ is closed under $*,(C, *, 1,0) \in \mathcal{R}_{0}$ due to Lemma 2.7 and the inclusion map $i_{C}: C \rightarrow A$ is a morphism in $\mathcal{R}_{0}$. Let us define a mapping $h: C \rightarrow A$ by $h(0)=0$ and $h(1)=h(d)=1$. Again, $h$ is a morphism in $\mathcal{R}_{0}$ and $m \circ h=m \circ i_{C}$. We conclude $h=i_{C}$, i.e., $d=i_{C}(d)=h(d)=1$. This yields $x=1 \wedge 1=1$.

Recall that a full subcategory $\mathcal{B}$ of a category $\mathcal{A}$ is said to be reflective in $\mathcal{A}$ if for each $\mathcal{A}$ object A there exists an $\mathcal{B}$-object $B_{A}$ and an $\mathcal{A}$-morphism $r_{A}: A \rightarrow B_{A}$ such that for each $\mathcal{A}$-morphism $h: A \rightarrow B$ to a $\mathcal{B}$-object $B$ there exists a unique $\mathcal{B}$-morphism $\bar{h}: B_{A} \rightarrow B$ with

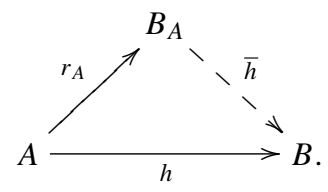

The pair $\left(B_{A}, r_{A}\right)$ is called the $\mathcal{B}$-reflection of $\mathcal{A}$. The morphism $r_{A}$ is called $\mathcal{B}$-reflection arrow. The corresponding functor $R: \mathcal{A} \rightarrow \mathcal{B}$, which is a left adjoint to the inclusion functor $I: \mathcal{B} \rightarrow \mathcal{A}$, is called reflector. The map $r_{A}$ is the unit of this adjunction.

We conclude from Lemmata 4.2 and 4.3.

Theorem 4.5 The category of Boolean algebras is a reflective subcategory of the category of bounded relatively pseudocomplemented posets and the reflector preserves monomorphisms. 
Let $\mathcal{A}$ be a category. An object $J$ of $\mathcal{A}$ is called injective if for every monomorphism $i: A \rightarrow B$ and every morphism $f: A \rightarrow J$ there exists a morphism $g: B \rightarrow J$ making the following diagram commute:

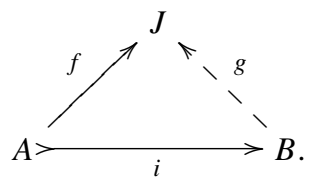

Theorem 4.6 $\mathbf{A}$ is injective in $\mathcal{R}_{0}$ if and only if $\mathbf{A}$ is a complete Boolean algebra.

Proof Assume first that $\mathbf{A}$ is injective in $\mathcal{R}_{0}$. From Theorem 3.1 we have an embedding $e_{\mathbf{A}}: \mathbf{A} \rightarrow \mathbf{D M}(\mathbf{A})$ of bounded relatively pseudocomplemented posets. From the injectivity of $\mathbf{A}$ we obtain the following commutative diagram:

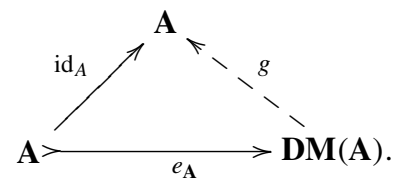

Since $g \circ e_{\mathbf{A}}=\operatorname{id}_{A}$ we have by [2, Lemma 2.1] that $\mathbf{A}$ is a complete lattice. To prove that $\mathbf{A}$ is a Boolean algebra we follow the lines of the proof of [2, Theorem 2.2]. It is enough to check that $x^{*}=0$ if and only $x=1$. Suppose that there is $a \in A$ such that $a^{*}=0$ and $a<1$. Then $\{0, a, 1\}$ is a bounded relatively pseudocomplemented subposet of $\mathbf{A}$ which is a chain. Let $\mathbf{C}$ be a maximal bounded relatively pseudocomplemented subposet of $\mathbf{A}$ which is a chain including $\{0, a, 1\}$ (which exists due to Proposition 2.7). Let $\mathbf{D}$ be the chain obtained by adding to $\mathbf{C}$ a new element $m$ such that $0<m<x$ for all $x \in C, x \neq 0$. Clearly, $\mathbf{D}$ is a bounded relatively pseudocomplemented poset equipped with a binary operation $*_{D}$ that coincides on $C$ with $*$ and $m *_{D} x=1=0 *_{D} m, m=x *_{D} m$ for all $x \in D, x \neq 0$, $m *_{D} 0=0$. We conclude that we have monomorphisms $i_{\mathbf{D}}: \mathbf{C} \rightarrow \mathbf{D}$ and $i_{\mathbf{A}}: \mathbf{C} \rightarrow \mathbf{A}$ of bounded relatively pseudocomplemented posets such that $i_{\mathbf{D}}(x)=i_{\mathbf{A}}(x)$ for all $x \in C$. From the injectivity of $\mathbf{A}$ we obtain the following commutative diagram:

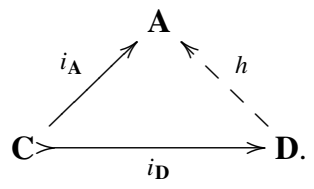

We see that $(h(D), *, 1,0)$ is a bounded relatively pseudocomplemented subposet of $\mathbf{A}$ which is a chain including $C$. Since $C$ was maximal we have that $h(m) \in C$. Let $x \in C$, $x \neq 0$. Since $m \leq x \in C \subseteq D$ we have that $h(m) \leq h(x)=x$. Let us compute:

$$
x * h(m)=h(x) * h(m)=h\left(x *_{D} m\right)=h(m) \leq h(a)=a<1 .
$$

We obtain that $h(m)<x$ for all $x \in C, x \neq 0$, i.e., $h(m)=0$. But $0=h(0)=h\left(m *_{D} 0\right)=$ $0 * 0=1$, a contradiction, i.e., there is no $a \in A$ such that $a^{*}=0$ and $a<1$. Hence $\mathbf{A}$ is a complete Boolean algebra. 
Now, let us verify that whenever $\mathbf{A}$ is a complete Boolean algebra then it is injective in $\mathcal{R}_{0}$. But this is evident since $\mathcal{R}_{0}$ is a full subcategory of the category of bounded Hilbert algebras and from [4, Theorem 5.5.2.] we know that complete Boolean algebras are injective objects in the category of bounded Hilbert algebras.

\section{Characterizing Quadruples}

In this section we introduce quadruples consisting of a Boolean algebra $\mathbf{B}$, a Hilbert algebra $\mathbf{D}$, a certain compatibility relation $\mathbf{C}$ between $\mathbf{B}$ and $\mathbf{D}$ and a join-preserving mapping $\varphi: \mathbf{B} \rightarrow$ Nuc $\mathbf{D}$ that preserves both the least and the greatest element. Our goal is to characterize every bounded Hilbert algebra and relatively pseudocomplemented poset by means of a quadruple. It is worth noticing that characterizing triples were introduced by C. C. Chen and G.Grätzer in [12] and intensively studied by T. Katriňák [23, 24] and his collaborators. For modular pseudocomplemented posets similar triples were introduced and investigated by the first author and R. Halaš in [8]. In our general case, triples do not produce a representation and hence we must use the so-called characterizing quadruples. Also, our results on characterizing Hilbert quadruples should be compared with the results by J. Cīrulis [13] on quasi-decompositions.

Let $\mathbf{A}=(A, *, 1,0)$ be a bounded Hilbert algebra. For $a \in B(\mathbf{A})$, put

$$
F_{a}:=\left\{x \in A \mid x^{* *}=a\right\} .
$$

The sets $F_{a}$ are equivalence classes of the Glivenko equivalence on $\mathbf{A}$. We say that an element $x \in A$ is dense if $x^{*}=0$, i.e., if and only if $x \in F_{1}$. We denote by $D(\mathbf{A})$ the set of all dense elements of $\mathbf{A}$. Note that $B(\mathbf{A}) \cap D(\mathbf{A})=\{1\}, D(\mathbf{A})$ is an implicative filter on $\mathbf{A}$ and $(D(\mathbf{A}), *, 1)$ is a Hilbert subalgebra of $\mathbf{A}$.

Let us consider the mapping $h_{a}: F_{a} \rightarrow D(\mathbf{A})$ given by $x \mapsto a * x, x \in F_{a}$ (note that $a * x \in D(\mathbf{A})$ due to Lemma 3.7). Every $x \in F_{a}$ is by Corollary 3.8 uniquely determined by $a * x$, namely $x=a \mathbb{\wedge}(a * x)$. Hence $h_{a}$ is an injective isotone mapping. Denote by $D_{a}$ the set $h_{a}\left(F_{a}\right)$. Then $h_{a}$ is by Lemma 1.4 (vi) an order isomorphism between $F_{a}$ and $D_{a}$ and $a * h_{a}(x)=h_{a}(x)$.

Note that

$$
\begin{aligned}
D_{a} & =\left\{a * x \in D(\mathbf{A}) \mid x=a \mathbb{\wedge}(a * x), x^{* *}=a, x \in A\right\} \\
& =\left\{d \in D(\mathbf{A}) \mid a \mathbb{\wedge} d \text { exists in } \mathbf{A} \text { and } a^{*} \leq d=a *(a \Uparrow d)\right\} \\
& =\{d \in D(\mathbf{A}) \mid a C d \text { and } a * d=d\}
\end{aligned}
$$

Namely, the first and the last equality are evident. Moreover, clearly $\{a * x \in D(\mathbf{A}) \mid x=$ $\left.a \mathbb{\wedge}(a * x), x^{* *}=a, x \in A\right\} \subseteq\left\{d \in D(\mathbf{A}) \mid a \mathbb{\wedge} d\right.$ exists in $\mathbf{A}$ and $\left.a^{*} \leq d=a *(a \mathbb{\wedge} d)\right\}$. Now, let $d \in D(\mathbf{A})$ such that $a \mathbb{\wedge} d$ exists in $\mathbf{A}$ and $a^{*} \leq d=a *(a \mathbb{\wedge} d)$. Put $x=a \mathbb{\wedge} d$. We have $d=a * x$. Let us check that $a=x^{* *}$. We know that $x=a \wedge d$ in the implicative envelope $\mathbf{S}(\mathbf{A})$ of $\mathbf{A}$. Hence (computing in $\mathbf{S}(\mathbf{A})$ ) $x^{* *}=a^{* *} \wedge_{\mathbf{S}(\mathbf{A})} d^{* *}=a \wedge_{\mathbf{S}(\mathbf{A})} 1=a$ since on implicative semilattices the operation ${ }^{* *}$ preserves finite meets.

By Lemma 3.7, any $x \in A$ is completely determined by a pair $(a, d)$, where $a=x^{* *} \in$ $B(\mathbf{A})$ and $d=a * x=a * d \in D_{a}$, namely $x=d \wedge a$, and every pair $(a, d)$ with $a \in B(\mathbf{A})$ and $d \in D_{a}$ determines in this way an element $x$ in $F_{a}$.

We have a compatibility relation $C(\mathbf{A}) \subseteq C \cap(B(\mathbf{A}) \times D(\mathbf{A}))$ between $B(\mathbf{A})$ and $D(\mathbf{A})$. Moreover, $C(\mathbf{A})=\bigcup_{a \in B(\mathbf{A})}\{a\} \times D_{a}=\left\{(a, d) \in C \cap(B(\mathbf{A}) \times D(\mathbf{A})) \mid u_{a}^{D(\mathbf{A})}(d)=d\right\}$. We also have $(\{1\} \times D(\mathbf{A})) \cup(B(\mathbf{A}) \times\{1\}) \subseteq C(\mathbf{A})$. 
Also, for any $a \in B(\mathbf{A})$ we have from Lemma 2.20 that $u_{a}^{D(\mathbf{A})}$ is a nucleus on $D(\mathbf{A})$ and we obtain a mapping $\bar{\varphi}: B(\mathbf{A}) \rightarrow \operatorname{Nuc} D(\mathbf{A})$ defined by $\bar{\varphi}(a)=u_{a^{*}}^{D(\mathbf{A})}$. Since $u_{a}^{D(\mathbf{A})}(d)=$ $a * d$ one readily sees that $\bar{\varphi}(0)=u_{1}^{D(\mathbf{A})}=\operatorname{id}_{D(\mathbf{A})}, \bar{\varphi}(1)=u_{0}^{D(\mathbf{A})}=\mathbf{1}$ and $a \leq b$ implies $b^{*} \leq a^{*}$ which entails $\bar{\varphi}(a)=u_{a^{*}}^{D(\mathbf{A})} \leq u_{b^{*}}^{D(\mathbf{A})}=\bar{\varphi}(b)$. Moreover, the compatibility relation $C(\mathbf{A})$ has by Lemma 3.9 the following property:

$$
(a, d),(b, e) \in C(\mathbf{A}) \Longrightarrow\left(a * b, \bar{\varphi}\left(a^{*}\right)(d * e)\right) \in C(\mathbf{A}) .
$$

To verify that $\bar{\varphi}$ is join-preserving note only that in implicative semilattices we have from [29, Lemma $2.1(\mathrm{v})]$ that $a *(b * d)=(a \wedge b) * d$. We may now safely work in $\mathrm{S}(\mathbf{A})$. Then we have $a *(b * d)=(a \wedge \mathrm{S}(\mathbf{A}) b) * d$. But if $a, b \in B(\mathbf{A})$ we obtain that $a \wedge_{\mathbf{S}(\mathbf{A})} b=a \wedge_{\mathbf{A}} b \in A$. Hence we have that $a *(b * d)=(a \wedge b) * d$, where $a, b \in B(\mathbf{A})$ and $d \in A$ is valid in any bounded Hilbert algebra. This in turn yields:

$\bar{\varphi}\left(a \vee_{B(\mathbf{A})} b\right)(d)=\left(a^{*} \wedge b^{*}\right) * d=a^{*} *\left(b^{*} * d\right)=\left(u_{a^{*}}^{D(\mathbf{A})} \circ u_{b^{*}}^{D(\mathbf{A})}\right)(d)=\left(\bar{\varphi}(a) \vee_{\mathrm{Nuc} D(\mathbf{A})} \bar{\varphi}(b)\right)(d)$.

Note that if $\mathbf{A}$ is a bounded implicative semilattice then our definition of a quadruple may be identified with that of a triple from [9] since the congruences $\Psi_{a}$ introduced in [9, Section 3.6] correspond uniquely to the nuclei $u_{a^{*}}^{D(\mathbf{A})}$ (both $\Psi_{a}$ and $u_{a^{*}}^{D(\mathbf{A})}$ are determined by the implicative filter $\left.U\left(a^{*}\right) \cap D(\mathbf{A})\right)$ and the relation $C(\mathbf{A})=\{(a, d) \in B(\mathbf{A}) \times D(\mathbf{A})$ | $\left.u_{a}^{D(\mathbf{A})}(d)=d\right\}$ is uniquely determined (hence there is no need for a quadruple).

In what follows we describe what properties $C(\mathbf{A})$ has to have in bounded pseudocomplemented Hilbert algebras.

Lemma 5.1 Let $\mathbf{A}=(A, *, 1,0)$ be a bounded pseudocomplemented Hilbert algebra, $(a, d),(b, e) \in C(\mathbf{A})$. Then the following holds:

$\left[(\forall(c, f) \in C(\mathbf{A}))\left(c *\left(a \wedge_{B(\mathbf{A})} b\right)=1=c *(f * d)=c *(f * e) \Longrightarrow c^{*}=1=f\right)\right] \Longrightarrow a \leq b^{*}$.

Proof The proof is a straightforward translation (using Lemma 3.9) of the fact that $L(a \Uparrow$

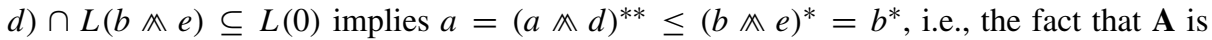
pseudocomplemented. Namely, $c *\left(a \wedge_{B(\mathbf{A})} b\right)=1$ means that $c \leq a, b, c *(f * d)=1$ means $c \leq f * d$ and $c *(f * e)=1$ means $c \leq f * e$. By Lemma 3.9 this is equivalent to

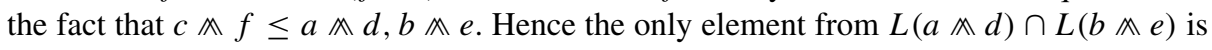
$0=0 \rtimes 1$.

Theorem 5.2 Let $\mathbf{A}=(A, *, 1,0)$ be a bounded Hilbert algebra. Then $\mathbf{A}$ is a pseudocomplemented poset if and only if the following condition is satisfied:

(RPP1) $\quad(\forall(a, d),(b, e) \in C(\mathbf{A}))\left(\left[(\forall(c, f) \in C(\mathbf{A}))\left(1=c *\left(a \wedge_{B(\mathbf{A})} b\right)=c *(f * d)=\right.\right.\right.$ $\left.\left.\left.c *(f * e) \Longrightarrow c^{*}=1=f\right)\right] \Longrightarrow a \leq b^{*}\right)$.

Proof $\Longrightarrow$ : This follows from Lemma 5.1.

$\Longleftarrow$ : Assume that $\mathbf{A}=(A, *, 1,0)$ is a bounded Hilbert algebra such that (RPP1) (defined in Theorem 5.5) holds. Let us check that $\mathbf{A}$ is pseudocomplemented. Let $x, y \in A$ such that $L(x) \cap L(y)=\{0\}=L(0)$. We can write $x=a \mathbb{A} d$ and $y=b \mathbb{A} e$ where $(a, d),(b, e) \in C(\mathbf{A})$. Clearly, for any $z=c \mathbb{\wedge} f \leq x, y,(c, f) \in C(\mathbf{A})$ we have $z=0$, i.e., $c=z^{* *}=0$ and $f=z^{* *} * z=0 * z=1$. Hence by (RPP1) we obtain $x \leq a \leq b^{*}=\left(b^{* *} \mathbb{\wedge} 1\right)^{*}=\left(b^{* *} \mathbb{\wedge} e^{* *}\right)^{*}=(b \mathbb{\wedge} e)^{*}=y^{*}$. Since A is a Hilbert 
algebra we have always from Lemma 2.6 that $L(y) \cap L\left(y^{*}\right) \subseteq L(0)=\{0\}$. Hence $y^{*}$ is a pseudocomplement of $y$ and $\mathbf{A}$ is pseudocomplemented.

Now we describe what properties $C(\mathbf{A})$ has to have in bounded relatively pseudocomplemented posets.

Lemma 5.3 Let $\mathbf{A}=(A, *, 1,0) \in \mathcal{R}_{0}, c, d, e \in D(\mathbf{A})$. Then the following holds:

$[(\forall(a, f) \in C(\mathbf{A}))(a *(f * c)=1=a *(f * d) \Longrightarrow a *(f * e)=1)] \Longrightarrow c *(d * e)=1$.

Proof The proof is a straightforward translation (using Lemma 3.9) of the fact that $L(c) \cap$ $L(d) \subseteq L(e)$ implies $c \leq d * e$, i.e., $(x=a \mathbb{\wedge} \leq c, d \Longrightarrow x \leq e)$ implies $c \leq d * e$.

Lemma 5.4 Let $\mathbf{A}=(A, *, 1,0) \in \mathcal{R}_{0}, b \in B(\mathbf{A}), d, e, f \in D(\mathbf{A})$. Then the following holds:

$[(\forall(a, g) \in C(\mathbf{A}))(a * b=a *(g * d)=1=a *(g * e) \Longrightarrow a *(g * f)=1)] \Longleftrightarrow$ $[(\forall(a, g) \in C(\mathbf{A}))(a *(g * d)=1=a *(g * e) \Longrightarrow a *(g *(b * f))=1)]$.

Proof The proof is a straightforward translation of the fact that $L(b) \cap L(d) \cap L(e) \subseteq L(f)$ if and only if $L(d) \cap L(e) \subseteq L(b * f)$. The implication from left to right is obtained by Theorem 3.1.

The preceding results yield the following characterization of bounded relatively pseudocomplemented posets:

Theorem 5.5 Let $\mathbf{A}=(A, *, 1,0)$ be a bounded Hilbert algebra. Then $\mathbf{A}$ is a bounded relatively pseudocomplemented poset if and only if the following conditions are satisfied:

(RPP1) $\quad(\forall(a, d),(b, e) \in C(\mathbf{A}))\left(\left[(\forall(c, f) \in C(\mathbf{A}))\left(1=c *\left(a \wedge_{B(\mathbf{A})} b\right)=c *(f * d)=\right.\right.\right.$ $\left.\left.\left.c *(f * e) \Longrightarrow c^{*}=1=f\right)\right] \Longrightarrow a \leq b^{*}\right)$,

(RPP2) $\quad(\forall c, d, e \in D(\mathbf{A}))([(\forall(a, f) \in C(\mathbf{A}))(a *(f * c)=1=a *(f * d) \Longrightarrow a *(f * e)$ $=1)] \Longrightarrow c *(d * e))=1)$, $(\forall b \in B(\mathbf{A}), d, e, f \in D(\mathbf{A}))$

(RPP3) $\quad[(\forall(a, g) \in C(\mathbf{A}))(a * b=a *(g * d)=1=a *(g * e) \Longrightarrow a *(g * f)=1)] \Longleftrightarrow$ $[(\forall(a, g) \in C(\mathbf{A}))(a *(g * d)=1=a *(g * e) \Longrightarrow a *(g *(b * f))=1)]$.

Proof $\Longrightarrow$ : This follows from Lemmata 5.1, 5.3 and 5.4.

$\Longleftarrow$ : Assume that $\mathbf{A}=(A, *, 1,0)$ is a bounded Hilbert algebra such that (RPP1), (RPP2) and (RPP3) hold. From Theorem 5.2 we know that $\mathbf{A}$ is pseudocomplemented. Moreover, from Theorem 22 in [30] we obtain that $\mathbf{D M}(\mathbf{A})$ with the operation * defined by $*(X)=X *\{0\}$ is pseudocomplemented as well.

Now, we have to verify that $\mathbf{A}$ is relatively pseudocomplemented. Let $x, y, z \in A$ such that $L(x) \cap L(y) \subseteq L(z)$. Since $\mathbf{D M}(\mathbf{A})$ is pseudocomplemented we obtain from [35,

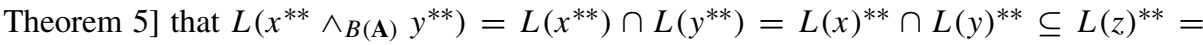
$L\left(z^{* *}\right)$. Hence $x^{* *} \wedge_{B(\mathbf{A})} y^{* *} \leq z^{* *}$ and from Corollary 3.4 we see that $x^{* *} \leq y^{* *} * z^{* *}$.

We need to show that $x \leq y * z$. Since $z=c \mathbb{A} f,(c, f) \in C(\mathbf{A})$ we have that $L(x) \cap L(y) \subseteq L(c)$ and $L(x) \cap L(y) \subseteq L(f)$. Also $y * z=y *(c \wedge f)=(y * c) \wedge(y * f)$. Hence it is enough to show that $x \leq y * c$ and $x \leq y * f$. We have $x=x^{* *} \mathbb{A} d$ and $y=y^{* *} \mathbb{\wedge} e, d, e \in D(\mathbf{A})$. 
Let us compute:

$$
y * c=\left(y^{* *} \mathbb{A} e\right) * c=y^{* *} *(e * c)=y^{* *} * c=y^{* *} * z^{* *} \geq x^{* *} \geq x .
$$

The second equality follows from [29, Lemma 2.1 (v)], the third one from Corollary 3.10. Now, we have

$$
L\left(x^{* *} \wedge_{B(\mathbf{A})} y^{* *}\right) \cap L(d) \cap L(e)=L\left(x^{* *} \bigwedge d\right) \cap L\left(y^{* *} \Uparrow e\right)=L(x) \cap L(y) \subseteq L(f) .
$$

But this is from (RPP3) equivalent to

$$
L(d) \cap L(e) \subseteq L\left(\left(x^{* *} \wedge_{B(\mathbf{A})} y^{* *}\right) * f\right) .
$$

We can now use (RPP2) to obtain that

$$
d \leq e *\left(\left(x^{* *} \wedge_{B(\mathbf{A})} y^{* *}\right) * f\right)=\left(x^{* *} \wedge_{\mathbf{A}} y^{* *}\right) *(e * f)=x^{* *} *\left(y^{* *} *(e * f)\right) .
$$

This yields

$$
x=x^{* *} 风 d \leq y^{* *} *(e * f)=\left(y^{* *} 风 e\right) * f=y * f .
$$

Hence $\mathbf{A}$ is relatively pseudocomplemented.

Definition 5.6 A characterizing quadruple is a quadruple $(\mathbf{B}, \mathbf{D}, \mathbf{C}, \varphi)$, where

(i) $\mathbf{B}=\left(B, *_{\mathbf{B}}, 1,0\right)$ is a Boolean algebra;

(ii) $\mathbf{D}=(D, * \mathbf{D}, 1)$ is a Hilbert algebra;

(iii) $\varphi: \mathbf{B} \rightarrow$ Nuc $\mathbf{D}$ is a join-preserving mapping that preserves both the least and the greatest element.

(iv) $\mathbf{C} \subseteq B \times D$ such that

$$
(a, d),(b, e) \in \mathbf{C} \Longrightarrow\left(a *_{\mathbf{B}} b, \varphi\left(a^{*}\right)\left(d *_{\mathbf{D}} e\right)\right) \in \mathbf{C}, \varphi\left(a^{*}\right)(d)=d
$$

and

$$
(\{1\} \times \mathbf{D}) \cup(\mathbf{B} \times\{1\}) \subseteq \mathbf{C} .
$$

A pcp-characterizing quadruple is a characterizing quadruple $(\mathbf{B}, \mathbf{D}, \mathbf{C}, \varphi)$ such that, for all $(a, d),(b, e) \in C(\mathbf{A})$, the following holds:

$$
\begin{aligned}
& {\left[( \forall ( c , f ) \in \mathbf { C } ) \left(\left(1=c *_{\mathbf{B}}\left(a \wedge_{\mathbf{B}} b\right) \text { and } 1=\varphi(c)\left(f *_{\mathbf{D}} d\right)=\varphi(c)\left(f *_{\mathbf{D}} e\right)\right)\right.\right.} \\
& \Longrightarrow(c=0 \text { and } 1=f))] \Longrightarrow a \leq b^{*} .
\end{aligned}
$$

An $r c p$-characterizing quadruple is a pcp-characterizing quadruple $(\mathbf{B}, \mathbf{D}, \mathbf{C}, \varphi)$ such that, for all $b \in B, d, e, f \in D$, the following holds:

$\left.\left[(\forall(a, g) \in \mathbf{C})\left(\varphi(a)\left(g *_{\mathbf{D}} d\right)=1=\varphi(a)\left(g *_{\mathbf{D}} e\right) \Longrightarrow \varphi(a)\left(g *_{\mathbf{D}} f\right)=1\right)\right] \Longrightarrow d *_{\mathbf{D}}\left(e *_{\mathbf{D}} f\right)\right)=1$

and

$\left[(\forall(a, g) \in \mathbf{C})\left(\left(a *_{\mathbf{B}} b=1\right.\right.\right.$ and $\left.\left.\left.\varphi(a)\left(g *_{\mathbf{D}} d\right)=1=\varphi(a)\left(g *_{\mathbf{D}} e\right)\right) \Longrightarrow \varphi(a)\left(g *_{\mathbf{D}} f\right)=1\right)\right]$ $\Longleftrightarrow\left[(\forall(a, g) \in \mathbf{C})\left(\varphi(a)\left(g *_{\mathbf{D}} d\right)=1=\varphi(a)\left(g *_{\mathbf{D}} e\right) \Longrightarrow \varphi(a)(g * \mathbf{D}(\varphi(b)(f)))=1\right)\right]$.

We conclude

Theorem 5.7 Let $\mathbf{A}=(A, *, 1,0)$ be a bounded Hilbert algebra. Then

(a) the quadruple $\mathbf{Q}=(B(\mathbf{A}), D(\mathbf{A}), C(\mathbf{A}), \bar{\varphi})$ is a characterizing quadruple;

(b) $\mathbf{Q}$ is a pcp-characterizing quadruple if $\mathbf{A}$ is pseudocomplemented;

(c) $\mathbf{Q}$ is an rcp-characterizing quadruple if $\mathbf{A}$ is relatively pseudocomplemented. 
We say that two characterizing quadruples $\left(\mathbf{B}_{1}, \mathbf{D}_{1}, \mathbf{C}_{1}, \varphi_{1}\right)$ and $\left(\mathbf{B}_{2}, \mathbf{D}_{2}, \mathbf{C}_{2}, \varphi_{2}\right)$ are isomorphic if there is an isomorphism $f$ of Boolean algebras from the Boolean algebra $\mathbf{B}_{1}$ to the Boolean algebra $\mathbf{B}_{2}$ and an isomorphism $g$ of Hilbert algebras from the Hilbert algebra $\mathbf{D}_{1}$ to the Hilbert algebra $\mathbf{D}_{2}$ such that $(f \times g)\left(\mathbf{C}_{1}\right)=\mathbf{C}_{2}$ and the following diagram

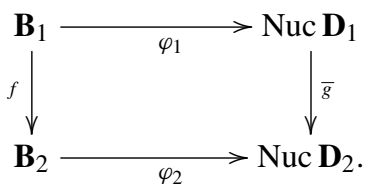

commutes, where $\bar{g}$ is the isomorphism of Nuc $\mathbf{D}_{1}$ to Nuc $\mathbf{D}_{2}$ assigning to each $j \in$ Nuc $\mathbf{D}_{1}$ the nucleus $g \circ j \circ g^{-1}$ on $\mathbf{D}_{2}$. Note that, for all $a \in B_{1}$ and all $d \in D_{2}, \varphi_{2}(f(a))(d)=$ $g\left(\varphi_{1}(a)\left(g^{-1}(d)\right)\right)$.

We are going to show that bounded Hilbert algebras are determined by their characterizing quadruples up to isomorphism.

Theorem 5.8 Two bounded Hilbert algebras are isomorphic if and only if their associated characterizing quadruples are isomorphic.

Proof It can be easily seen that if $h$ is an isomorphism of bounded Hilbert algebras from the bounded Hilbert algebra $\mathbf{A}_{1}$ to the bounded Hilbert $\mathbf{A}_{2}$ then its restrictions $f=h \mid B(\mathbf{A})$ and $g=h \mid D(\mathbf{A})$ are the isomorphisms in question.

Conversely, let us suppose that the characterizing quadruples $\left(B\left(\mathbf{A}_{1}\right), D\left(\mathbf{A}_{1}\right)\right.$, $\left.C\left(\mathbf{A}_{1}\right), \bar{\varphi}_{1}\right)$ and $\left(B\left(\mathbf{A}_{2}\right), D\left(\mathbf{A}_{2}\right), C\left(\mathbf{A}_{2}\right), \bar{\varphi}_{2}\right)$ are isomorphic, with corresponding isomorphisms $f$ and $g$. Hence, for all $a \in B\left(\mathbf{A}_{1}\right)$ and all $d \in D\left(\mathbf{A}_{2}\right), f(a) * d=g(a *$ $\left.g^{-1}(d)\right)$.

Let $\mathbf{S}\left(\mathbf{A}_{i}\right)$ be the implicative envelope of $\mathbf{A}_{i}$. Then $\mathbf{S}\left(B\left(\mathbf{A}_{i}\right)\right)=B\left(\mathbf{A}_{i}\right)$ and $\mathbf{S}\left(D\left(\mathbf{A}_{i}\right)\right)=$ $D\left(\mathbf{S}\left(\mathbf{A}_{i}\right)\right), i=1,2$. Hence we obtain characterizing quadruples $\mathbf{Q}_{1}=\left(B\left(\mathbf{A}_{1}\right), \mathbf{S}\left(D\left(\mathbf{A}_{1}\right)\right)\right.$, $\left.B\left(\mathbf{A}_{1}\right) \times_{\psi_{1}} \mathrm{~S}\left(D\left(\mathbf{A}_{1}\right)\right), \bar{\psi}_{1}\right)$ and $\mathbf{Q}_{2}=\left(B\left(\mathbf{A}_{2}\right), \mathrm{S}\left(D\left(\mathbf{A}_{2}\right)\right), B\left(\mathbf{A}_{2}\right) \times_{\psi_{2}} \mathrm{~S}\left(D\left(\mathbf{A}_{2}\right)\right), \bar{\psi}_{2}\right)$ where $\overline{\psi_{i}}\left(a_{i}\right)=\mathrm{S}\left(u_{a_{i}^{*}}^{D\left(\mathbf{A}_{i}\right)}\right)=u_{a_{i}^{*}}^{D\left(\mathrm{~S}\left(\mathbf{A}_{i}\right)\right)}, a_{i} \in A_{i}$ by Proposition 2.19 and $B\left(\mathbf{A}_{1}\right) \times \psi_{1}$ $\mathrm{S}\left(D\left(\mathbf{A}_{1}\right)\right)=\left\{\left(a_{i}, d_{i}\right) \in B\left(\mathbf{A}_{i}\right) \times \mathrm{S}\left(D\left(\mathbf{A}_{i}\right)\right) \mid u_{a_{i}}^{\mathrm{S}\left(D\left(\mathbf{A}_{i}\right)\right)}\left(d_{i}\right)=d_{i}\right\}, i=1,2$. These charac-

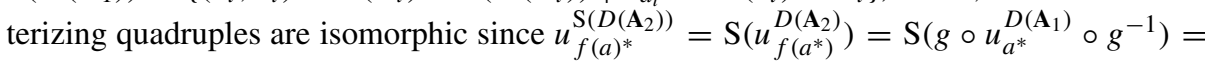
$\mathrm{S}(g) \circ \mathrm{S}\left(u_{a^{*}}^{D\left(\mathbf{A}_{1}\right)}\right) \circ \mathrm{S}(g)^{-1}=\mathrm{S}(g) \circ u_{a^{*}}^{\mathrm{S}\left(D\left(\mathbf{A}_{1}\right)\right)} \circ \mathrm{S}(g)^{-1}$ with corresponding isomorphisms $f$ and $\mathbf{S}(g)$.

Define a mapping $h: \mathbf{S}\left(\mathbf{A}_{1}\right) \rightarrow \mathbf{S}\left(\mathbf{A}_{2}\right)$ via

$$
h(x):=f\left(x^{* *}\right) \wedge \mathrm{S}(g)\left(x^{* *} * x\right)
$$

for all $x \in \mathrm{S}\left(\mathbf{A}_{1}\right)$.

Since $\mathbf{S}\left(\mathbf{A}_{1}\right)$ and $\mathbf{S}\left(\mathbf{A}_{2}\right)$ are pseudocomplemented semilattices satisfying assumptions of [9, Theorem 3.6.1 and Theorem 4.4.1] we obtain that $h$ is an order isomorphism preserving $*, f=h \mid B\left(\mathbf{A}_{1}\right)$ and $\mathrm{S}(g)=h \mid D\left(\mathbf{A}_{1}\right)$.

In what follows we put $\tilde{g}=\mathrm{S}(g)$. Then the fact that the characterizing quadruples $\mathbf{Q}_{1}$ and $\mathbf{Q}_{2}$ are isomorphic is equivalent to the following condition:

$$
\tilde{g}(a * d)=f(a) * \widetilde{g}(d)
$$

for all $a \in B\left(\mathbf{A}_{1}\right)$ and $d \in \mathrm{S}\left(D\left(\mathbf{A}_{1}\right)\right)$. 
Note first that, for all $x \in \mathrm{S}\left(A_{1}\right), h(x)^{* *} * h(x)=h\left(x^{* *} * x\right)$. Since $x=x^{* *} \wedge\left(x^{* *} * x\right)$ and $h$ preserves arbitrary meets and ${ }^{*}$ we have:

$$
\begin{aligned}
h(x)^{* *} * h(x) & =h(x)^{* *} * h\left(x^{* *} \wedge\left(x^{* *} * x\right)\right)=h(x)^{* *} *\left(h\left(x^{* *}\right) \wedge h\left(x^{* *} * x\right)\right) \\
& =\left(h(x)^{* *} * h\left(x^{* *}\right)\right) \wedge\left(h(x)^{* *} * h\left(x^{* *} * x\right)\right)=1 \wedge\left(h(x)^{* *} * h\left(x^{* *} * x\right)\right) \\
& =h\left(x^{* *}\right) * h\left(x^{* *} * x\right) .
\end{aligned}
$$

Let us verify that $h$ preserves the operation $*$. Assume that $x, z \in \mathrm{S}\left(A_{1}\right)$. Then we have

$$
\begin{aligned}
h(x) * h(z) & =\left(h(x)^{* *} * h(z)^{* *}\right) \wedge\left(h(x)^{* *} *\left[\left(h(x)^{* *} * h(x)\right) *\left(h(z)^{* *} * h(z)\right)\right]\right) \\
& =\left(h\left(x^{* *}\right) * h\left(z^{* *}\right)\right) \wedge\left(h\left(x^{* *}\right) *\left[h\left(x^{* *} * x\right) * h\left(z^{* *} * z\right)\right]\right) \\
& =\left(f\left(x^{* *}\right) * f\left(z^{* *}\right)\right) \wedge\left(f\left(x^{* *}\right) *\left[\widetilde{g}\left(x^{* *} * x\right) * \widetilde{g}\left(z^{* *} * z\right)\right]\right) \\
& =\left(f\left(x^{* *} * z^{* *}\right)\right) \wedge\left(f\left(x^{* *}\right) *\left[\widetilde{g}\left(\left(x^{* *} * x\right) *\left(z^{* *} * z\right)\right)\right]\right) \\
& =\left(h\left(x^{* *} * z^{* *}\right)\right) \wedge \widetilde{g}\left(x^{* *} *\left[\left(x^{* *} * x\right) *\left(z^{* *} * z\right)\right]\right) \\
& =\left(h\left(x^{* *} * z^{* *}\right)\right) \wedge h\left(x^{* *} *\left[\left(x^{* *} * x\right) *\left(z^{* *} * z\right)\right]\right) \\
& =h\left(\left(x^{* *} * z^{* *}\right) \wedge\left(x^{* *} *\left[\left(x^{* *} * x\right) *\left(z^{* *} * z\right)\right]\right)\right) \\
& =h(x * z) .
\end{aligned}
$$

The first and the last equalities follow from Lemma 3.9 (ii), the second one from the fact that $h$ preserves ${ }^{*}$ and that $h(x)^{* *} * h(x)=h\left(x^{* *} * x\right)$, the third one from the fact that $f=h \mid B\left(\mathbf{A}_{1}\right)$ and $\tilde{g}=h \mid D\left(\mathbf{A}_{1}\right)$, the fourth one since $f$ and $\widetilde{g}$ preserve $*$, the fifth equality follows again from $f=h \mid B\left(\mathbf{A}_{1}\right)$ and the isomorphism of $\mathbf{Q}_{1}$ and $\mathbf{Q}_{2}$, the sixth one from $\tilde{g}=h \mid D\left(\mathbf{A}_{1}\right)$, the seventh equality holds because $h$ preserves finite meets.

Let $x \in A_{1}$. Then from Lemma 3.7 we have that $x=x^{* *} \wedge\left(x^{* *} * x\right)$, i.e., $\left(x^{* *}, x^{* *} * x\right) \in$ $C\left(\mathbf{A}_{1}\right)$. We conclude $\left(f\left(x^{* *}\right), g\left(x^{* *} * x\right)\right) \in C\left(\mathbf{A}_{2}\right)$. Then there is an element $y \in A_{2}$ such that $y=f\left(x^{* *}\right) \wedge g\left(x^{* *} * x\right)$. Hence $y^{* *}=f\left(x^{* *}\right)^{* *} \wedge g\left(x^{* *} * x\right)^{* *}=f\left(x^{* *}\right)^{* *} \wedge 1=$ $f\left(x^{* * *}\right)=f\left(x^{* *}\right)$. Since $f\left(x^{* *}\right) * g\left(x^{* *} * x\right)=g\left(x^{* *} *\left(x^{* *} * x\right)\right)=g\left(x^{* *} * x\right)$ and $y=y^{* *} \wedge\left(y^{* *} * y\right)$ we have again from Lemma 3.7 that $g\left(x^{* *} * x\right)=y^{* *} * y$. This yields that $h\left(A_{1}\right) \subseteq A_{2}$.

Now, let $y \in A_{2}$. Then $y=y^{* *} \mathbb{\wedge}\left(y^{* *} * y\right)$ and $\left(y^{* *}, y^{* *} * y\right) \in C\left(\mathbf{A}_{2}\right)$. Hence there are $a \in B\left(\mathbf{A}_{1}\right)$ and $d \in D\left(\mathbf{A}_{1}\right)$ such that $f(a)=y^{* *}, g(d)=y^{* *} * y$ and $(a, d) \in C\left(\mathbf{A}_{1}\right)$. Put $x=a \mathbb{\wedge} d \in A_{1}$. Then $h(x)=h(a \mathbb{\wedge} d)=h(a) \mathbb{\wedge} h(d)=f(a) \mathbb{\wedge} g(d)=y$.

Since $h$ is injective we obtain that $h \mid A_{1}$ is a bijective mapping from $A_{1}$ to $A_{2}$ that preserves $*, 1$ and 0 , i.e., it is an isomorphism of bounded Hilbert algebras.

Corollary 5.9 Two (relatively) pseudocomplemented bounded Hilbert algebras are isomorphic if and only if their associated pcp-characterizing (rcp-characterizing) quadruples are isomorphic.

Theorem 5.10 For any characterizing quadruple $\mathbf{Q}=(\mathbf{B}, \mathbf{D}, \mathbf{C}, \varphi)$, there exists a bounded Hilbert algebra $\mathbf{A}_{\mathbf{Q}}$ whose associated characterizing quadruple $\mathcal{Q}\left(\mathbf{A}_{\mathbf{Q}}\right)=\left(B\left(\mathbf{A}_{\mathbf{Q}}\right)\right.$, $\left.D\left(\mathbf{A}_{\mathbf{Q}}\right), C\left(\mathbf{A}_{\mathbf{Q}}\right), \bar{\varphi}_{\mathbf{Q}}\right)$, is isomorphic to $\mathbf{Q}$.

Proof Let $\widetilde{\mathbf{Q}}=(\mathbf{B}, \widetilde{\mathbf{D}}, \widetilde{\mathbf{C}}, \widetilde{\varphi})$ be a quadruple defined as follows: We put $\widetilde{\mathbf{D}}=\mathrm{S}(\mathbf{D}), \widetilde{\mathbf{C}}=$ $\mathbf{B} \times{ }_{\varphi} \widetilde{\mathbf{D}}=\left\{(a, d) \in B \times \mathbf{S}(\mathbf{D}) \mid \widetilde{\varphi}\left(a^{*}\right)(d)=d\right\}$ and $\tilde{\varphi}(a)=\mathrm{S}(\varphi(a))$ for all $a \in B$. We want to show that $\widetilde{\mathbf{Q}}=(\mathbf{B}, \widetilde{\mathbf{D}}, \widetilde{\mathbf{C}}, \widetilde{\varphi})$ is a characterizing quadruple. Conditions (i) and (ii) of Definition 5.6 are readily satisfied. Let us check condition (iii). Assume first that $a, b \in B$. We compute:

$$
\widetilde{\varphi}\left(a \vee_{\mathbf{B}} b\right)=\mathrm{S}\left(\varphi\left(a \vee_{\mathbf{B}} b\right)\right)=\mathrm{S}(\varphi(a) \circ \varphi(b))=\mathrm{S}(\varphi(a)) \circ \mathrm{S}(\varphi(b))=\widetilde{\varphi}(a) \vee_{\widetilde{\mathbf{D}}} \widetilde{\varphi}(b)
$$


Moreover, $\widetilde{\varphi}(0)=\mathrm{S}(\varphi(0))=\mathrm{S}\left(\operatorname{id}_{\mathbf{D}}\right)=\mathrm{id}_{\mathrm{S}(\mathbf{D})}=\mathrm{id}_{\widetilde{\mathbf{D}}}$ and $\widetilde{\varphi}(1)=\mathrm{S}(\varphi(1))=\mathrm{S}\left(\mathbf{1}_{\mathbf{D}}\right)=$ $\mathbf{1}_{\mathrm{S}(\mathbf{D})}=\mathbf{1}_{\widetilde{\mathbf{D}}}$. To verify (iv) assume that $(a, c),(b, d) \in \widetilde{\mathbf{C}}$. We have to check that

$$
\widetilde{\varphi}\left((a * b)^{*}\right)\left(\widetilde{\varphi}\left(a^{*}\right)(c * \widetilde{\mathbf{D}} d)\right)=\widetilde{\varphi}\left(a^{*}\right)(c * \widetilde{\mathbf{D}} d) .
$$

We compute:

$$
\begin{aligned}
\widetilde{\varphi}\left(a^{*}\right)(c * \widetilde{\mathbf{D}} d) & \leq \widetilde{\varphi}\left((a * b)^{*}\right)\left(\widetilde{\varphi}\left(a^{*}\right)(c * \widetilde{\mathbf{D}} d)\right)=\widetilde{\varphi}\left(a^{*}\right)\left(\widetilde{\varphi}\left((a * b)^{*}\right)(c * \widetilde{\mathbf{D}} d)\right) \\
& =\widetilde{\varphi}\left(a^{*}\right)\left(\widetilde{\varphi}\left((a * b)^{*}\right)(c) * \widetilde{\mathbf{D}} \widetilde{\varphi}\left((a * b)^{*}\right)(d)\right) \\
& \leq \widetilde{\varphi}\left(a^{*}\right)\left(\widetilde{\varphi}(0)(c) * \widetilde{\mathbf{D}} \widetilde{\varphi}\left(b^{*}\right)(d)\right)=\widetilde{\varphi}\left(a^{*}\right)(c * \widetilde{\mathbf{D}} d) .
\end{aligned}
$$

The first inequality follows from the fact that $\widetilde{\varphi}\left((a * b)^{*}\right)$ is a nucleus, the next equality follows by interchangeability of the composition of nuclei (the composition of two nuclei is their join). Then we use the fact that $\tilde{\varphi}\left((a * b)^{*}\right)$ preserves $* \widetilde{\mathbf{D}}$. The second inequality follows from the fact that $\left.\tilde{\varphi}(0)(c) \leq \widetilde{\varphi}\left((a * b)^{*}\right)(c), \widetilde{\varphi}\left((a * b)^{*}\right)\right)(d) \leq \widetilde{\varphi}\left(b^{*}\right)(d)$ and the properties of $* \widetilde{\mathbf{D}}$ (Lemma $1.4(\mathrm{v}),(\mathrm{vi}))$. The last equality follows from the fact that $\widetilde{\varphi}(0)=\mathrm{id} \widetilde{\mathbf{D}}$ and that $(b, d) \in \widetilde{\mathbf{C}}\left(\widetilde{\varphi}\left(b^{*}\right)(d)=d\right)$. The remaining parts of (iv) are evident.

Now one can repeat the construction from [29, Theorem 5.2] of a bounded implicative semilattice $\widetilde{\mathbf{A}}_{\mathbf{Q}}$ whose associated characterizing triple corresponds uniquely to our characterizing quadruple $\widetilde{\mathbf{Q}}=(\mathbf{B}, \widetilde{\mathbf{D}}, \widetilde{\mathbf{C}}, \widetilde{\varphi})$. Note that an admissible function $f$ of $[29$, Theorem 5.2] is in our sense defined as $f(a, d)=\widetilde{\varphi}\left(a^{*}\right)(d)$. We only describe here $\widetilde{\mathbf{A}}_{\mathbf{Q}}$.

We put $\widetilde{\mathbf{A}}_{\mathbf{Q}}=\widetilde{\mathbf{C}}$, and for elements $(a, d),(b, e) \in \widetilde{\mathbf{C}}$ let

$$
(a, d) \wedge_{\tilde{\mathbf{A}}_{\mathbf{Q}}}(b, e)=\left(a \wedge_{\mathbf{B}} b, \widetilde{\varphi}\left(\left(a \wedge_{\mathbf{B}} b\right)^{*}\right)\left(d \wedge_{\widetilde{\mathbf{D}}} e\right)\right)
$$

and

$$
(a, d) * \widetilde{\mathbf{A}}_{\mathbf{Q}}(b, e)=\left(a * \mathbf{B} b, \widetilde{\varphi}\left(a^{*}\right)\left(d *_{\widetilde{\mathbf{D}}} e\right)\right) .
$$

Then $\left(\widetilde{\mathbf{A}}_{\mathbf{Q}}, \wedge \widetilde{\mathbf{A}}_{\mathbf{Q}}, * \widetilde{\mathbf{A}}_{\mathbf{Q}},(1,1),(0,1)\right)$ is an implicative semilattice with the associated characterizing quadruple $\left(B\left(\tilde{\mathbf{A}}_{\mathbf{Q}}\right), D\left(\widetilde{\mathbf{A}}_{\mathbf{Q}}\right), C\left(\widetilde{\mathbf{A}}_{\mathbf{Q}}\right), \bar{\varphi}_{\mathbf{Q}}\right)$. Here $B\left(\widetilde{\mathbf{A}}_{\mathbf{Q}}\right)=\{(a, 1) \mid a \in B\}$, $(a, 1) *_{B\left(\tilde{\mathbf{A}}_{\mathbf{Q}}\right)}(b, 1)=\left(a *_{\mathbf{B}} b, \widetilde{\varphi}\left(a^{*}\right)\left(1 *_{\widetilde{\mathbf{D}}} 1\right)\right)=\left(a *_{\mathbf{B}} b, 1\right)$. Hence the mapping $\mathrm{id}_{\mathbf{B}} \mathbf{1}$ given by the prescription $a \mapsto(a, 1)$ is an isomorphim of Boolean algebras. Similarly, $D\left(\widetilde{\mathbf{A}}_{\mathbf{Q}}\right)=\{(a, 1) \mid a \in D\},(1, d) *_{D\left(\widetilde{\mathbf{A}}_{\mathbf{Q}}\right)}(1, e)=\left(1 *_{\mathbf{B}} 1, \widetilde{\varphi}(0)\left(d *_{\widetilde{\mathbf{D}}} e\right)\right)=\left(1, d *_{\widetilde{\mathbf{D}}} e\right)$. Therefore the mapping $\mathrm{id}_{\widetilde{\mathbf{D}}}^{\mathbf{1}}$ given by the prescription $d \mapsto(1, d)$ is an isomorphism of Hilbert algebras.

It remains to check that $\operatorname{id}_{\widetilde{\mathbf{D}}}^{\mathbf{1}}(\widetilde{\varphi}(a)(d))=\bar{\varphi}\left(\operatorname{id}_{\mathbf{B}}^{\mathbf{1}}(a)\right)\left(\operatorname{id}_{\widetilde{\mathbf{D}}}^{\mathbf{1}}(d)\right)$ for all $a \in \mathbf{B}, d \in \widetilde{\mathbf{D}}$. Let us compute:

$$
\begin{aligned}
\operatorname{id}_{\widetilde{\mathbf{D}}} & \mathbf{1}(\widetilde{\varphi}(a)(d))=(1, \widetilde{\varphi}(a)(d))=\left(a *_{\mathbf{B}} 1, \widetilde{\varphi}(a)\left(1 *_{\widetilde{\mathbf{D}}} d\right)\right) \\
& =\left(a^{*}, 1\right) *_{\widetilde{\mathbf{A}}_{\mathbf{Q}}}(1, d)=\bar{\varphi}\left(\operatorname{id}_{\mathbf{B}}^{\mathbf{1}}(a)\right)\left(\operatorname{id}_{\widetilde{\mathbf{D}}}^{\mathbf{1}}(d)\right) .
\end{aligned}
$$

We conclude that the characterizing quadruples $\widetilde{\mathbf{Q}}=(\mathbf{B}, \widetilde{\mathbf{D}}, \widetilde{\mathbf{C}}, \widetilde{\varphi})$ and $\left(B\left(\widetilde{\mathbf{A}}_{\mathbf{Q}}\right), D\left(\widetilde{\mathbf{A}}_{\mathbf{Q}}\right)\right.$, $\left.C\left(\widetilde{\mathbf{A}}_{\mathbf{Q}}\right), \bar{\varphi} \widetilde{\mathbf{Q}}\right)$ are isomorphic.

Now, let us put $\mathbf{A}_{\mathbf{Q}}=\mathbf{C} \subseteq \widetilde{\mathbf{C}}$. Let $(a, d),(b, e) \in \mathbf{C}$. Since $\mathbf{Q}$ is a characterizing quadruple we can use condition (iv) of its definition that says that $(a, d) * \widetilde{\mathbf{A}}_{\mathbf{Q}}(b, e)=$ $\left(a *_{\mathbf{B}} b, \widetilde{\varphi}\left(a^{*}\right)(d * \widetilde{\mathbf{D}} e)\right) \in \mathbf{C}$. Clearly $(0,1),(1,1) \in \mathbf{C}$. Hence $\left(\mathbf{A}_{\mathbf{Q}}, *_{\mathbf{A}_{\mathbf{Q}}},(1,1),(0,1)\right)$ is a Hilbert subalgebra of $\widetilde{\mathbf{A}}_{\mathbf{Q}}$ where $* \mathbf{A}_{\mathbf{Q}}$ is a restriction of $* \widetilde{\mathbf{A}}_{\mathbf{Q}}$.

Clearly $B\left(\mathbf{A}_{\mathbf{Q}}\right)=\mathbf{B} \times\{1\}, D\left(\mathbf{A}_{\mathbf{Q}}\right)=\{1\} \times \mathbf{D}$ and $C\left(\mathbf{A}_{\mathbf{Q}}\right)=\left\{((a, 1),(1, d)) \in B\left(\mathbf{A}_{\mathbf{Q}}\right) \times\right.$ $D\left(\mathbf{A}_{\mathbf{Q}}\right) \mid(a, 1) C(1, d)$ in $\mathbf{A}_{\mathbf{Q}}$, and $\left.(a, 1) * \mathbf{A}_{\mathbf{Q}}(1, d)=(1, d)\right\}=\{((a, 1),(1, d)) \mid(a, d) \in$ $\mathbf{C}$. We know that the mapping $\operatorname{id}_{\mathbf{B}}^{\mathbf{1}}: \mathbf{B} \rightarrow B\left(\mathbf{A}_{\mathbf{Q}}\right)$ given by the prescription $a \mapsto(a, 1)$ is an isomorphim of Boolean algebras. Similarly, the mapping $\mathrm{id}_{\mathbf{D}}^{\mathbf{1}}: \mathbf{D} \rightarrow D\left(\mathbf{A}_{\mathbf{Q}}\right)$ given by the 
prescription $d \mapsto(1, d)$ is an isomorphism of Hilbert algebras, $\overline{\mathrm{id}_{\mathbf{D}}^{\mathbf{1}}}$ is the isomorphism of Nuc $\mathbf{D}$ to Nuc $D\left(\widetilde{\mathbf{A}}_{\mathbf{Q}}\right)$ assigning to each $j \in \operatorname{Nuc} \mathbf{D}$ the nucleus $\operatorname{id}_{\mathbf{D}}^{\mathbf{1}} \circ j \circ\left(\operatorname{id}_{\mathbf{D}}^{\mathbf{1}}\right)^{-1}$ on $D\left(\widetilde{\mathbf{A}}_{\mathbf{Q}}\right)$, and $\left(\operatorname{id}_{\mathbf{B}}^{\mathbf{1}} \times \operatorname{id}_{\mathbf{D}}^{\mathbf{1}}\right)(\mathbf{C})=C\left(\mathbf{A}_{\mathbf{Q}}\right)$.

Moreover,

$$
\begin{aligned}
\bar{\varphi}_{\mathbf{Q}}\left(\operatorname{id}_{\mathbf{B}}^{\mathbf{1}}(a)\right)((1, d)) & =\bar{\varphi}_{\mathbf{Q}}((a, 1))((1, d))=u_{(a, 1)^{*}}^{D\left(\mathbf{A}_{\mathbf{Q}}\right)}((1, d))=\left(a^{*}, 1\right) * \mathbf{A}_{\mathbf{Q}}(1, d) \\
& =\left(a^{*}, 1\right) * \widetilde{\mathbf{A}}_{\mathbf{Q}}(1, d)=\left(a^{*} * \mathbf{B} 1, \widetilde{\varphi}\left(a^{* *}\right)(1 * \widetilde{\mathbf{D}} d)\right)=(1, \varphi(a)(d)) \\
& =\operatorname{id}_{\mathbf{D}}^{\mathbf{1}}(\varphi(a)(d))=\left(\operatorname{id}_{\mathbf{D}}^{\mathbf{1}} \circ \varphi(a) \circ\left(\operatorname{id}_{\mathbf{D}}^{\mathbf{1}}\right)^{-1}\right)((1, d))=\overline{\operatorname{id}_{\mathbf{D}}^{\mathbf{1}}}(\varphi(a))((1, d))
\end{aligned}
$$

for all $a \in \mathbf{B},(1, d) \in D\left(\mathbf{A}_{\mathbf{Q}}\right)$. Hence $\overline{\mathrm{id}_{\mathbf{D}}^{\mathbf{1}}} \circ \varphi=\bar{\varphi}_{\mathbf{Q}} \circ \mathrm{id}_{\mathbf{B}}^{\mathbf{1}}$. It is easy to see that $\mathcal{Q}\left(\mathbf{A}_{\mathbf{Q}}\right)=$ $\left(B\left(\mathbf{A}_{\mathbf{Q}}\right), D\left(\mathbf{A}_{\mathbf{Q}}\right), C\left(\mathbf{A}_{\mathbf{Q}}\right), \bar{\varphi}_{\mathbf{Q}}\right)$ is a characterizing quadruple which is isomorphic to $\mathbf{Q}$ via isomorphisms $\operatorname{id}_{\mathbf{B}}^{\mathbf{1}}$ and $\operatorname{id}_{\mathbf{D}}^{\mathbf{1}}$.

Corollary 5.11 (a) For any pcp-characterizing quadruple $\mathbf{Q}$, there exists a bounded pseudocomplemented Hilbert algebra $\mathbf{A}_{\mathbf{Q}}$ whose associated pcp-characterizing quadruple $\mathcal{Q}\left(\mathbf{A}_{\mathbf{Q}}\right)$ is isomorphic to $\mathbf{Q}$.

(b) For any rcp-characterizing quadruple $\mathbf{Q}$, there exists a bounded relatively pseudocomplemented poset $\mathbf{A}_{\mathbf{Q}}$ whose associated rcp-characterizing quadruple $\mathcal{Q}\left(\mathbf{A}_{\mathbf{Q}}\right)$ is isomorphic to $\mathbf{Q}$.

Proof (a): From the proof of Theorem 5.10 we obtain a bounded Hilbert algebra $\mathbf{A}_{\mathbf{Q}}$. Evidently, it satisfies condition (RPP1) from Theorem 5.2. Hence it is pseudocomplemented. The remaining parts then follow from Theorem 5.10.

(b): From the proof of part (a) we obtain a bounded pseudocomplemented Hilbert algebra $\mathbf{A}_{\mathbf{Q}}$ such that $\mathcal{Q}\left(\mathbf{A}_{\mathbf{Q}}\right)$ is isomorphic to $\mathbf{Q}$. Evidently, it satisfies conditions (RPP1)(RPP3) from Theorem 5.5. Hence it is relatively pseudocomplemented.

We are going to show that, given a Boolean algebra $\mathbf{B}$, a Hilbert algebra $\mathbf{D}$ and an action $\varphi$ of $\mathbf{B}$ on $\mathbf{D}$, the relation $\mathbf{C}$ can be introduced in various ways to yield various Hilbert algebras with the corresponding quadruplets. In Example 5.12 we show for which relation $\mathbf{C}$ the resulting Hilbert algebra is the smallest or the greatest one. This also proves that the triple $(\mathbf{B}, \mathbf{D}, \varphi)$ is not sufficient for a characterization of the resulting Hilbert algebra.

Example 5.12 Let $\mathbf{B}=\left(B, *_{\mathbf{B}}, 1,0\right)$ be a Boolean algebra and $\mathbf{D}=\left(D, *_{\mathbf{D}}, 1\right)$ be a Hilbert algebra. Let $\varphi: \mathbf{B} \rightarrow$ Nuc $\mathbf{D}$ be a join-preserving mapping that preserves both the least and the greatest element.

(a) We put $\mathbf{C}=(\{1\} \times \mathbf{D}) \cup(\mathbf{B} \times\{1\})$. Let us check condition (iv) from Definition 5.6. Assume that $(a, d),(b, e) \in \mathbf{C}$. We have

$$
\left(a *_{\mathbf{B}} b, \varphi\left(a^{*}\right)\left(d *_{\mathbf{D}} e\right)\right)= \begin{cases}\left(a *_{\mathbf{B}} b, \varphi\left(a^{*}\right)\left(1 *_{\mathbf{D}} 1\right)\right)=\left(a *_{\mathbf{B}} b, 1\right) \in \mathbf{C} \text { if } d=e=1, \\ \left(1 *_{\mathbf{B}} 1, \varphi(0)\left(d *_{\mathbf{D}} e\right)\right)=\left(1, d *_{\mathbf{D}} e\right) \in \mathbf{C} \quad \text { if } a=b=1, \\ \left(1 *_{\mathbf{B}} b, \varphi(0)\left(d *_{\mathbf{D}} 1\right)\right)=(b, 1) \in \mathbf{C} \quad \text { if } a=1 \text { and } e=1, \\ \left(a *_{\mathbf{B}} 1, \varphi(0)\left(1 *_{\mathbf{D}} e\right)\right)=(1, \varphi(0)(e)) \in \mathbf{C} \text { if } b=1 \text { and } d=1,\end{cases}
$$

and

$$
\varphi\left(a^{*}\right)(d)= \begin{cases}\varphi(0)(d)=d & \text { if } a=1 \\ \varphi\left(a^{*}\right)(1)=1=d & \text { if } d=1 .\end{cases}
$$


Hence $(a, d),(b, e) \in \mathbf{C}$ implies $\left(a *_{\mathbf{B}} b, \bar{\varphi}\left(a^{*}\right)\left(d *_{\mathbf{D}} e\right)\right) \in \mathbf{C}$ and $\bar{\varphi}\left(a^{*}\right)(d)=d$. We conclude that $\mathbf{Q}=(\mathbf{B}, \mathbf{D}, \mathbf{C}, \varphi)$ is a characterizing quadruple and the corresponding Hilbert algebra will be $\left(\mathbf{A}_{\mathbf{Q}}, *_{\mathbf{A}_{\mathbf{Q}}},(1,1),(0,1)\right)$ according to Theorem 5.10. Evidently, this is the smallest Hilbert algebra with prescribed Boolean algebra of closed elements isomorphic to $\mathbf{B}$ and prescribed Hilbert algebra of dense elements isomorphic to $\mathbf{D}$ and the construction does not depend on $\varphi$.

(b) We put $\mathbf{C}=\left\{(a, d) \in B \times D \mid \varphi\left(a^{*}\right)(d)=d\right\}$. Again, let us verify condition (iv) from Definition 5.6. We have $\varphi\left(1^{*}\right)(d)=\varphi(0)(d)=d$ and $\varphi\left(a^{*}\right)(1)=1$, i.e., $(\{1\} \times \mathbf{D}) \cup(\mathbf{B} \times\{1\}) \subseteq \mathbf{C}$. Assume that $(a, d),(b, e) \in \mathbf{C}$. By the same arguments as in the proof of Theorem 5.10 we obtain

$$
\varphi\left(\left(a *_{\mathbf{B}} b\right)^{*}\right)\left(\varphi\left(a^{*}\right)\left(d *_{\mathbf{D}} e\right)\right)=\varphi\left(a^{*}\right)\left(d *_{\mathbf{D}} e .\right)
$$

But this is equivalent to $\left(a *_{\mathbf{B}} b, \bar{\varphi}\left(a^{*}\right)\left(d *_{\mathbf{D}} e\right)\right) \in \mathbf{C}$. The last condition of (iv) from Definition 5.6 is satisfied by the definition of $\mathbf{C}$ and $\mathbf{Q}=(\mathbf{B}, \mathbf{D}, \mathbf{C}, \varphi)$ is a characterizing quadruple. Now, the corresponding Hilbert algebra $\left(\mathbf{A}_{\mathbf{Q}}, *_{\mathbf{A}_{\mathbf{Q}}},(1,1),(0,1)\right)$ according to Theorem 5.10 is the greatest Hilbert algebra with prescribed Boolean algebra of closed elements isomorphic to $\mathbf{B}$, prescribed Hilbert algebra of dense elements isomorphic to $\mathbf{D}$ and chosen action of $\mathbf{B}$ on $\mathbf{D}$.

Remark 5.13 The usual motivation for various triple constructions has been the desire for reduction of algebras under consideration to simpler, or better known algebraic structures. In our construction we follow the idea of W.C. Nemitz [29] for constructing bounded implicative semilattices having a given Boolean algebra for closed algebra, and a given implicative semilattice for dense filter. As in [29] we work with an action of the Boolean algebra on dense elements but instead of making a quotient of the cartesian product of the Boolean algebra with the dense filter we directly specify possible pairs of elements of our Boolean algebra and dense elements. The reason is that our represented structure does not have meets and congruences do not always induce relatively pseudocomplemented posets, i.e., we need to be more demanding in other conditions.

Acknowledgements Open access funding provided by Austrian Science Fund (FWF). We would like to express our gratitude to the anonymous reviewers whose thorough and detailed comments lead to a considerable improvement of this paper and helped to enhance the results of this paper in a substantial manner.

Open Access This article is distributed under the terms of the Creative Commons Attribution 4.0 International License (http://creativecommons.org/licenses/by/4.0/), which permits unrestricted use, distribution, and reproduction in any medium, provided you give appropriate credit to the original author(s) and the source, provide a link to the Creative Commons license, and indicate if changes were made.

\section{References}

1. Balbes, R.: On free pseudo-complemented and relatively pseudo-complemented semi-lattices. Fund. Math. 78, 119-131 (1973)

2. Balbes, R., Horn, A.: Injective and projective Heyting algebras. Trans. Amer. Math. Soc. 148, 549-559 (1970)

3. Bușneag, D.: A note on deductive systems of a Hilbert algebra. Kobe J. Math. 2, 29-35 (1985)

4. Bușneag, D.: Categories of Algebraic Logic. Ed. Academiei Române 2006. ISBN 978973-27-1381-5

5. Celani, S.A., Jansana, R.: On the free implicative semilattice extension of a Hilbert algebra. Math. Log. Q. 58, 188-207 (2012) 
6. Chajda, I.: Relatively pseudocomplemented directoids. Comment. Math. Univ. Carolin. 50, 349-357 (2009)

7. Chajda, I.: Pseudocomplemented and Stone posets. Acta Univ. Palacki. Olomuc. Fac. Rerum Natur. Math. 51, 29-34 (2012)

8. Chajda, I., Halaš, R.: Characterizing triplets for modular pseudocomplemented ordered sets. Math. Slovaca 50, 513-524 (2000)

9. Chajda, I., Halaš, R., Kühr, J.: Semilattice Structures. Heldermann, Lemgo 2007. ISBN 978-3-88538230-0

10. Chajda, I., Länger, H.: Relatively pseudocomplemented posets. Math. Bohem. 143, 89-97 (2018)

11. Chajda, I., Snášel, V.: Congruences in ordered sets. Math. Bohem. 123, 95-100 (1998)

12. Chen, C.C., Grätzer, G.: Stone lattices. II. Structure theorems. Canad. J. Math. 21, 895-903 (1969)

13. Cīrulis, J.: Multipliers, closure endomorphisms and quasi-decompositions of a Hilbert algebra. Contrib. Gen. Algebra 16, 25-34 (2005)

14. Cīrulis, J.: Hilbert algebras as implicative partial semilattices. Cent. Eur. J. Math. 5, 264-279 (2007)

15. Cīrulis, J.: Adjoint semilattice and minimal Brouwerian extensions of a Hilbert algebra. Acta Univ. Palacki. Olomuc., Fac. rer. nat. Mathematica 51, 41-51 (2012)

16. Cīrulis, J.: Lattice of closure endomorphisms of a Hilbert algebra. arXiv:1701.03902 (2017)

17. Curry, H.B.: Lecons de Logique Algébrique. (French) (Collection De Logique Mathematique. Ser. A. II.) Paris: Gauthier-Villars, Louvain,: E. Nauwelaerts (1952)

18. Curry, H.B.: Foundations of Mathematical Logic. Dover, New York (1977)

19. Diego, A.: Sobre Algebras de Hilbert Notas De Logica Mat, vol. 12. Inst. Mat. Univ. Nac. del Sur, Bahia Blanca (1965)

20. Diego, A.: Sur les Algèbres de Hilbert. (French). Gauthier-Villars, E. Nauwelaerts, Paris-Louvain (1966)

21. Frink, O.: Pseudo-complements in semi-lattices. Duke Math. J. 29, 505-514 (1962)

22. Ghiță, M.: Some categorical properties of Hilbert algebras. Annals of University of Craiova. Math. Comp. Sci. Ser. 36, 95-104 (2009)

23. Katriňák, T.: Bemerkung über pseudokomplementären halbgeordneten Mengen. (German) Acta Fac. Rer. Nat. Univ. Comenianae, Math. 19, 181-185 (1968)

24. Katriňák, T.: Pseudokomplementäre Halbverbände. (German) Mat. Čas., Slovensk. Akad. Vied 18, 121143 (1968)

25. Köhler, P.: Brouwerian semilattices. Transactions of AMS 268, 103-126 (1981)

26. Macnab, D.S.: Modal operators on Heyting algebras. Algebra Universalis 12, 5-29 (1981)

27. Marsden, E.L.: Compatible elements in implicative models. J. Philos. Log. 1, 156-161 (1972)

28. Marsden, E.L.: A note on implicative models. Notre Dame J. Formal Log. 14, 139-144 (1972)

29. Nemitz, W.C.: Implicative semi-lattices. Trans. Amer. Math. Soc. 117, 128-142 (1965)

30. Niederle, J.: On pseudocomplemented and Stone ordered sets. Order 18, 161-170 (2001)

31. Pawar, Y.S.: Implicative posets. Bull. Calcutta Math. Soc. 85, 381-384 (1993)

32. Rasiowa, H.: An Algebraic Approach to Non-Classical Logics. PWN, North-Holland Publ. Co., Warszawa, Amsterdam, London (1974)

33. Rudeanu, S.: On relatively pseudocomplemented posets and Hilbert algebras. An. Stiint. Univ.Iasi, N. Ser., Ia, Suppl. 31, 74-77 (1985)

34. Rudeanu, S.: On the Glivenko-Frink theorem for Hilbert algebras. An. Univ. Craiova, Ser. Mat. Inf. 34, 73-78 (2007)

35. Venkatanarasimhan, P.V.: Pseudo-complements in posets. Proc. Am. Math. Soc. 28, 9-17 (1971)

Publisher's Note Springer Nature remains neutral with regard to jurisdictional claims in published maps and institutional affiliations. 